\title{
A Hierarchical and Contextual Model for Aerial Image Parsing
}

\author{
Jake Porway • Qiongchen Wang • Song Chun Zhu
}

Received: 28 July 2008 / Accepted: 20 October 2009 / Published online: 3 November 2009

(c) The Author(s) 2009. This article is published with open access at Springerlink.com

\begin{abstract}
In this paper we present a hierarchical and contextual model for aerial image understanding. Our model organizes objects (cars, roofs, roads, trees, parking lots) in aerial scenes into hierarchical groups whose appearances and configurations are determined by statistical constraints (e.g. relative position, relative scale, etc.). Our hierarchy is a nonrecursive grammar for objects in aerial images comprised of layers of nodes that can each decompose into a number of different configurations. This allows us to generate and recognize a vast number of scenes with relatively few rules. We present a minimax entropy framework for learning the statistical constraints between objects and show that this learned context allows us to rule out unlikely scene configurations and hallucinate undetected objects during inference. A similar algorithm was proposed for texture synthesis (Zhu et al. in Int. J. Comput. Vis. 2:107-126, 1998) but didn't incorporate hierarchical information. We use a range of different bottom-up detectors (AdaBoost, TextonBoost, Compositional Boosting (Freund and Schapire in J. Comput. Syst. Sci. 55, 1997; Shotton et al. in Proceedings of the European Conference on Computer Vision, pp. 1-15, 2006; $\mathrm{Wu}$ et al. in Proceedings of the IEEE Conference on Computer Vision and Pattern Recognition, pp. 1-8, 2007)) to propose locations of objects in new aerial images and employ a cluster sampling algorithm (C4 (Porway and Zhu, 2009)) to choose the subset of detections that best explains the im-
\end{abstract}

J. Porway $(\bowtie) \cdot$ Q. Wang · S.C. Zhu

Department of Statistics, University of California, Los Angeles,

USA

e-mail: jakeporway@gmail.com

Q. Wang · S.C. Zhu

Lotus Hill Institute for Computer Vision and Information Science,

Ezhou, China age according to our learned prior model. The $\mathrm{C} 4$ algorithm can quickly and efficiently switch between alternate competing sub-solutions, for example whether an image patch is better explained by a parking lot with cars or by a building with vents. We also show that our model can predict the locations of objects our detectors missed. We conclude by presenting parsed aerial images and experimental results showing that our cluster sampling and top-down prediction algorithms use the learned contextual cues from our model to improve detection results over traditional bottom-up detectors alone.

Keywords Hierarchical models · Scene-level context . Statistical learning - Image understanding - Aerial images . Swendsen-Wang clustering $\cdot$ Bayesian inference

\section{Introduction}

\subsection{Objectives and Motivation}

Aerial image understanding is an important field of research for tackling the problems of automated navigation, large scale 3D scene construction, and object tracking for use in event detection. Most of the tasks using aerial images need or would benefit from a full explanation of the scene, consisting of the locations and scales of detected objects and their relationships to one another. Being able to identify objects of many different types and understand their relationships to one another gives a deeper understanding of the data and allows subsequent algorithms to make smarter decisions faster.

There are difficulties in aerial image parsing that do not arise in more restricted recognition tasks. Three major obstacles to modeling aerial images are, 


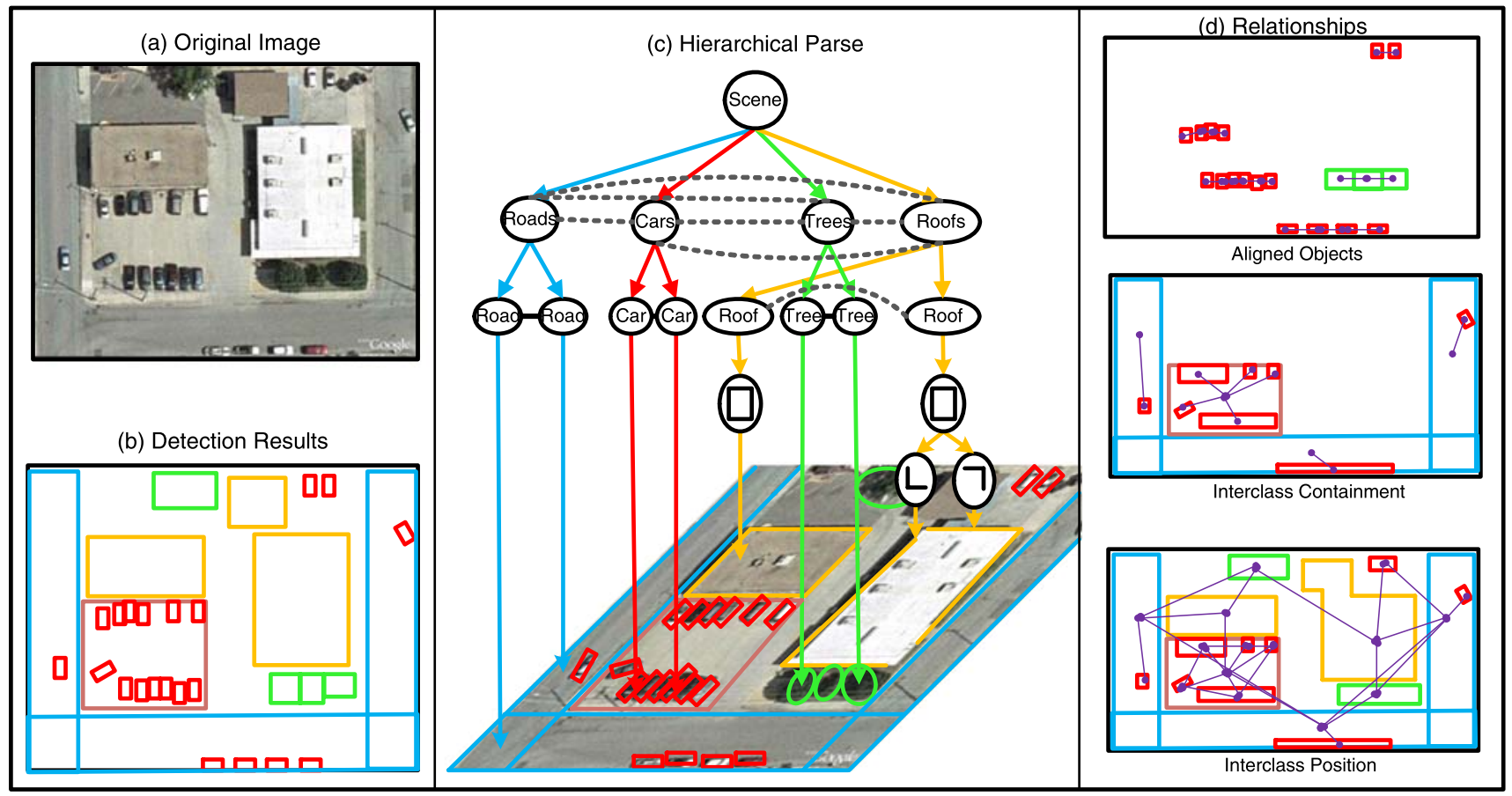

Fig. 1 An example of a hierarchically parsed aerial image. (a) The original image. (b) A flat configuration of objects in the scene. (c) A hierarchical parse graph of the scene. (d) Three typical contextual relationships and the objects related by them

1. Highly Variant Configurations: Objects in aerial images can appear at many different locations, scales, and orientations in the image, creating a vast number of possible configurations. There may also be hundreds of objects present, making it infeasible to create a rigid model that can enumerate every possible spatial layout.

2. Multi-resolution: Objects in aerial images appear at a number of resolutions, from small cars about 10 pixels wide to massive roofs more than 800 pixels long. There is no single feature or detector that is likely to perform well across all categories and all sizes of object.

3. Coupling Constraints: Certain objects frequently appear together under certain constraints, for example cars often appear in parking lots. When performing inference to find the best explanation of the scene, one must make sure the explanation adheres to this strong coupling. If an image patch is explained by a roof with vents on top of it, changing that explanation to a parking lot with cars in it requires updating all of the objects involved at once. Single site sampling methods are insufficient for this task, as they would add/subtract single objects to and from the current explanation one at a time, each of which would be a very lowprobability state on its own. We need some way to switch all the involved objects at once, removing the roof from the explanation while at the same time adding the cars and parking lot to the explanation together.

To motivate our solution to the problems above, let us look at an example of parsing an aerial image. Our goal is to simultaneously detect objects in images and organize them into a hierarchical contextual representation for the scene, i.e. which objects are grouped together and how they're related to one another. Figure 1 shows an example of what a parsed scene looks like. Figure 1(b) shows a flat configuration of detected objects in a typical aerial image. In Fig. 1(c) these objects have been grouped hierarchically - nearby cars are aligned in rows, trees form treelines, and proximal buildings form city blocks, for example. This hierarchical representation explains the image at many resolutions and abstracts the objects into loosely related groups. It also models the number of groups we see in each image and the number of objects we observe in each group. Figure 2 shows a visualization of this representation.

Even though objects in groups don't appear in rigid formations the way the parts of a single object might, we recognize certain spatial and appearance constraints that they must obey. For example, we would never expect to see two cars on top of one another or a building smaller than a tree. These constraints between objects and groups are represented as horizontal lines between nodes in Fig. 1(c). Figure 1(d) shows examples of which objects could be related by certain constraints. The first panel shows which cars and trees are aligned in a straight line. The second panel shows which objects contain other objects, and the last panel shows that almost all objects in this example are related by their relative position. We want our model to automatically learn which constraints between which objects are sufficient to 
Fig. 2 The 3-level contextual hierarchy. The scene is broken down into groups of objects of one of five categories, which are in turn broken down into individual objects of the same type. The thick vertical arrows between the scene and groups and between the groups and objects indicate that these nodes can decompose into a variable number of children. Objects are represented by detectors, some of which (roofs, roads) are hierarchical themselves. The horizontal lines between nodes at the same level represent statistical constraints on the nodes' appearance

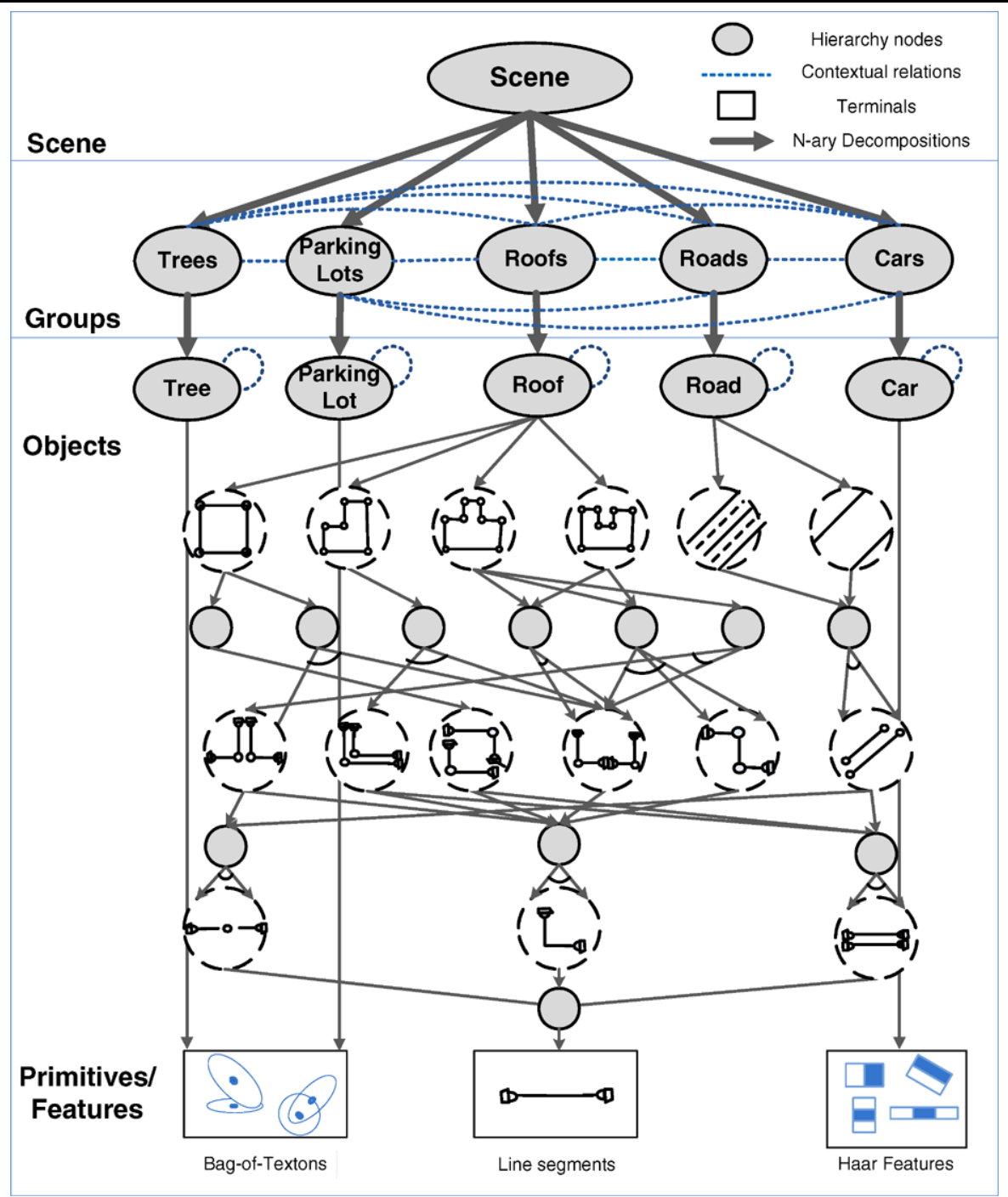

represent the scene. If objects in a scene violate constraints that we expect to see or don't meet enough of them, then that interpretation of the scene will have a low probability. Such constraints would also rule out invalid object detections (e.g. cars on top of trees).

\subsection{Major Contributions}

We present a 3-layer hierarchy with embedded contextual constraints along with a 3-stage inference algorithm to solve the problems above and capture the natural hierarchical and contextual nature of aerial images.

1. Hierarchical and Contextual Model: To handle the large structural variations of aerial images we model scenes as groups of like objects, such as cars aligned in rows or roofs clustered into city blocks (see Fig. 2). This abstracts the scene into loosely related neighborhoods. We then add statistical constraints within and between these groups to constrain their relative appearances, such as how close to- gether they are or what size they are. We achieve this by embedding Markov random fields (MRFs) into a hierarchical grammar model. Our grammar model begins at a root scene node that then probabilistically decomposes into a number of group nodes, such as $n$ groups of cars or $m$ groups of roofs. Each of these groups can in turn decompose into a number of single nodes, such as $j$ cars in one group, $k$ cars in another. Alone this hierarchy only captures the frequency of objects, however, so we add contextual relationships via MRFs on the neighborhoods within each group and between groups. In this way we create a statistical model that uses a small set of decomposition rules to generate variable number of objects whose appearances are constrained by statistical relationships.

2. Automatic Learning of Context by Relationship Selection: Creating the constraints for our model by hand is infeasible due to the huge number of potential constraints that could exist, so we present an algorithm to automatically add statistical constraints to the hierarchy in a minimax entropy 
framework. This minimax entropy technique was used previously in texture modeling (Zhu et al. 1998) and on more general graphical models (Wainwright and Jordan 2008) but we are now applying it to a hierarchical model. We seek to model the true distribution of aerial images, $f$, with our learned distribution, $p$, by iteratively matching feature statistics between $f$ and $p$. This matching entails extracting features from a set of observed data (which follows $f$ ) and adjusting our model $p$ such that it reproduces the statistics of these features. This learning method automatically selects the most important feature statistics to match and ignores low-information features. This allows us to add only relevant relationships from a large dictionary of potential constraints. By the end of this process, samples from $p$ appear similar to true samples from $f$ along the learned dimensions.

3. Flexible Detectors for Multiple Object Categories: The 3-level hierarchy terminates at object nodes, below which we may plug in any detectors that we like for each object type. Large textured regions, such as grass, trees, parking lots, water, and dirt, are detected using a Bag-of-Textons classifier. In this work, small patch-like objects, like cars, are detected using Haar features and AdaBoost. Roofs and roads, which have many different colors and shapes, are detected using edges as features and a compositional algorithm called Compositional Boosting (Wu et al. 2007) for detection. Compositional Boosting is itself a hierarchical detector and groups edges into larger and larger structures if they meet certain appearance constraints. In our work, edges detected in the image are grouped into corners, Tjunctions, long lines, etc. that are then grouped into polygons. These polygonal boundaries are stronger indicators of a roof's presence than color or texture are and can model many different roof shapes.

4. Top-Down Bayesian Inference with Cluster Sampling and Prediction: To handle the coupling constraints that appear in aerial images we use a sampling algorithm inspired by Swendsen-Wang clustering (Barbu and Zhu 2005; Swendsen and Wang 1987). Swendsen-Wang cluster sampling was introduced to sample the Potts model more effectively by updating a cluster of sites at once instead of just a single site. Our variant of this algorithm, named Clustering via Cooperative and Competitive Constraints (C4) (Porway and Zhu 2009), updates multiple clusters at once, allowing us to move very rapidly in the solution space. The clusters represent competing explanations of the scene. For example, a patch explained by a parking lot with cars may be better explained by a single roof. To swap these explanations, we couple the cars with the parking lot and switch the whole cluster with the roof in a single step. This process results in an explanation of the scene with very few false positives. We also use the hierarchical nature of our top-down model to propose new objects our object detectors may have missed, thus increasing the number of true positives in our final result.
It bears noting that our training process uses hand-labeled images as input. This requires having a human identify objects of interest in images and label their boundaries. Thankfully only the boundaries of the objects need to be labeled, regardless of the number of contextual relationships being learned by the algorithm.

\subsection{Related Work}

Our work is related to two subfields of computer vision: aerial image parsing and hierarchical object recognition. In the aerial image parsing literature it is very rare to find work that detects multiple types of objects simultaneously. Most work focuses on detecting just one type of object, rarely using context or hierarchy to model the whole scene. In the object recognition category we often see complex hierarchical and contextual models. However, these models are often designed for rigid objects where appearance constraints are fairly constant between instances of the object.

Much work has been done on identifying single objects in aerial images, such as rooftops (Maloof et al. 2003; Vestri and Devernay 2001; Wei and Prinet 2005), cars (Li et al. 2005; Zhao and Nevatia 2001), or roads (Nicolas et al. 2000). In these cases context plays little role, as single objects are detected without taking the support of surrounding objects into consideration. These works use similar object detectors to those we use, though they almost exclusively use one detector without considering the support from multiple detectors. These detectors include AdaBoost (Freund and Schapire 1997; Viola and Jones 2001), Bag of Words (Berg et al. 2007; Sivic et al. 2005) and TextonBoost (Shotton et al. 2006).

Some aerial imaging works incorporate context and/or multiple object category detection into the same framework. SIGMA (Matsuyama and Hang 1990), a knowledge-based "expert system" for aerial images, was an attempt to model rule-based spatial relationships between objects. Unfortunately, as in much of the computer science based AI work of that time, relationships were often hardcoded and thus not generally extensible. On a smaller scale, Moissinac identified roads and city blocks in urban scenes using local context rules to decide how roads connect and how blocks should appear (Moissinac et al. 1994). Hinz used positional relations to determine the likely positions of roads in aerial images (Hinz and Baumgartner 2000). A recent approach for parsing images of outdoor scenes by Berg et al. (2007) also seeks to model images as collections of regions that obey positional and relational constraints. As far as we know, however, these models require a good deal of hand-tuning and hardcoded logic in order to encode the relevant constraints. SIGMA relied on experts to identify relationships of interest to model, Moissinac knew exactly the domain he was working with (handdrawn maps) and designed relationships 
accordingly, and Berg et al. used domain knowledge of the objects they wanted to identify to design contextual cues. Our model improves upon this shortcoming by employing a minimax entropy learning framework to automatically select significant relationships from a bank of potential relationships that can be designed to work on many domains of data without constant user input.

Our model borrows concepts from grammars in natural language, so we would be remiss not to credit the large and growing field of object recognition using hierarchies and context in non-aerial image recognition. Growing out of the early grammar work by Fu and Ohta $(1981,1985)$ on grammars for line drawings, much recent work has attended to the process of learning compositional object structures that employ local context to resolve ambiguities. The constellation model (Weber et al. 2000), pictorial structures (Felzenszwalb and Huttenlocher 2005; Fischler and Elschlager 1973), and patch hierarchies (Ullman et al. 2001) all use learned statistical constraints to model the relative position of object parts to some reference frame. Higher level compositional structures for object categories ( $\mathrm{Li}$ and Perona 2005; Todorovic and Ahuja 2006) have shown great performance on object detection and localization tasks and can even be learned from unlabeled images, while work on rulebased models of shape have shown the power of statistical composition (Keselman and Dickinson 2001; Siddiqi et al. 1999). Some of these models can express the general relationships present between shared object parts of the same category, a very useful trait for generalization.

In later sections we define our model as an exponential model over a graphical structure. We employ minimax entropy techniques seen in Zhu et al. (1998) to learn the parameters of this model and pursue its contextual relationships. However, we must acknowledge the huge number of contributions made in modeling graphical models as exponential families from all walks of machine learning and computer vision. We refer to the tutorial by Jordan and Wainwright on this topic (Wainwright and Jordan 2008) on similar uses of maximum entropy for modeling distributions on graphical models.

More general grammars for full scene modeling have developed recently (Chen et al. 2006; Han and Zhu 2005; Jin and Geman 2006; Tu and Zhu 2002; Zhu et al. 2008; Zhu and Mumford 2006). The work in Han and Zhu (2005), Tu and Zhu (2002) seek to explain an entire image by parsing it hierarchically into constituent regions and objects, while Chen et al. (2006), Jin and Geman (2006), Zhu et al. (2008), Zhu and Mumford (2006) focus more on single objects. These models borrow closely from models used in natural language processing, and express structural and appearance variation as the result of production rules. Hierarchical models for objects that include scene-level constraints have been presented in Singhal et al. (2003), Sudderth et al.
(2005), which are very similar in spirit to our model. The contextual constraints, however, tend to strictly be relative position constraints. Moreover, while this entire corpus of work is hugely important for object recognition, many of the contributions here rely on the fairly constant arrangement of a relatively fixed number of parts in objects. The variation in part appearance, frequency, and location for a motorcycle is far lower than that of the arrangement of cars and roofs in a city scene.

In the field of natural language processing, much progress has also been made on Unification-Based Grammars (UBG), which seek to model sentences by augmenting a tree structure with additional features, such as pairwise frequencies of words or attributes of the sentence as a whole (e.g. number of direct objects) (Johnson et al. 1999). This work is very related to ours in that the researchers seek to extend a tree structure to include relationship constraints. While much successful work has been done using UBG's, the authors of this article have yet to encounter a straightforward way for automatically adding constraints to these models, much less adding constraints proportional to their importance. UBG's are mostly seen in the field of natural language processing, where the input data types are much more constrained than in vision and can thus be labeled and tagged more easily by hand. Our method provides a technique for automatically selecting the most representative pairwise features to be added to the model using minimax entropy.

We present an overview of our contextual hierarchy, learning algorithm, and Bayesian inference process in Sect. 2. This is followed by the formulation of our hierarchical and contextual model in Sect. 3 and by a description of the learning process in Sect. 4 . We show that sampled aerial images drawn from our model are composed similarly to aerial images that we trained from in Sects. 5 and 6. We finally present a Bayesian framework for a three-stage inference algorithm in Sect. 7 before closing with experiments in Sect. 8 and conclusions in Sect. 9.

\section{Overview}

In this section we give an overview of our 3-layer hierarchy and the learning algorithm for adding context to our representation. We also describe our Bayesian inference algorithm, a 3-step process that first detects objects using different detectors, then uses cluster sampling to remove false positives from our explanation before using top-down prediction to detect any objects our explanation is missing.

\subsection{Hierarchical and Contextual Representation}

Figure 2 shows our 3-level hierarchy. It consists of nodes divided into a root scene node, group nodes, and object nodes. 
Group nodes are collections of the same type of object, such as blocks of roofs or lines of cars, while object nodes are the single objects within each group. Below this level is the object representation level, which may be hierarchical in and of itself, as in the case of roofs and roads in our example, or may terminate at a one-layer representation for the object. The top 3-levels are representation agnostic, however, so we will put off a discussion of object detection and representation until Sect. 7.1. The thick arrow edges between the scene node and group nodes and between the group nodes and the object nodes indicate that a varying number of each group node may be present, and the number of object nodes they are comprised of can vary as well. The hierarchy is similar to a grammar, where the scene node decomposes into a variable number of object groups, which in turn decompose into a variable number of objects. This captures the loose, variable nature of aerial images with just a few compact rules. If we were to write these expansions in a grammar format, we would write

$$
\begin{aligned}
\text { Scene } \rightarrow & \left(\text { Roads }^{*}\right) \cup\left(\text { Roofs }^{*}\right) \cup\left(\text { Trees }^{*}\right) \cup\left(\text { Parking Lots }^{*}\right) \\
& \cup\left(\text { Cars }^{*}\right) \\
\text { Roads } \rightarrow & \text { Road }^{*} \\
\text { Roofs } \rightarrow & \text { Roof }^{*} \\
\text { Trees } \rightarrow & \text { Trees } * \\
\text { Parking Lots } \rightarrow \text { Parking Lot } & \\
\text { Cars } \rightarrow & \text { Car }^{*} .
\end{aligned}
$$

Here we're using " "*" in the regular expression sense, meaning 0 or more of an object. One could rewrite the "**" operator by enumerating all cases, as in

\section{Roads}

$$
\rightarrow \emptyset \mid \text { Road } \mid(\text { Road })(\text { Road }) \mid(\text { Road })(\text { Road })(\text { Road }) \mid \ldots
$$

On its own the hierarchy simply captures the number of object groups and objects in the scene. We also add statistical constraints between objects to ensure that their appearance and configuration obey certain statistical properties, such as relative scale, relative position, etc. These statistical constraints are represented as dotted horizontal lines in Fig. 2 and can be any measurable statistic between some non-empty set of object nodes.

\subsection{Minimax Entropy Learning}

We use a minimax entropy learning framework for automatically adding the horizontal constraints to the model during learning. The algorithm first gathers feature statistics $M_{f}$ across a set of aerial images. For clarity we will use the term "relationship statistic" to mean feature statistic for the remainder of the paper. For example, a relationship statistic could be the distribution of the relative scale between every pair of cars in every image in our training set. The responses for each relationship statistic (e.g. relative scale between cars, relative position between roofs, etc.) are then modeled by a continuous parameterized distribution or just as 1-D histograms. We assume we can draw samples from our current model, which begins as just the hierarchy of object groups with no constraints. These samples will be aerial images themselves. We can gather the same relationship statistics $M_{p}$ across our sampled images, again using the example of measuring the relative scale between every pair of cars in every image we sampled. We find the statistic $r_{i}$ that differs the most between $M_{f}$ and $M_{p}$, thus indicating its importance. We add this constraint to our model and then repeat the procedure until no more relationship statistics differ significantly. By the end of this process, the most important relationship statistics over samples from our model will match the most important relationship statistics over true aerial images. Figure 3 visualizes this process, and the results of learning the model are presented in Sect. 6.

\subsection{Top-Down and Bottom-Up Bayesian Inference with the 3-Level Hierarchy}

In this section we give an overview of the 3-stage algorithm for parsing new aerial images. In the first stage we detect objects in the image using bottom-up detectors. The next stage then prunes false positives using cluster sampling, followed by the third stage, which predicts missing objects based on our current explanation of the scene.

1. Bottom-Up Detection: In the first phase we collect bottom-up proposals for each object category of interest. The detectors used to find each of these objects can be any off-the-shelf detector and may detect many false positives in the scene. We use Bags of textons and edges, along with a number of boosting methods, to detect objects from multiple categories at multiple scales. Textured objects, like parking lots and trees, are detected at the pixel-level, while structured objects, like roofs and roads, are composed from edges in the image. By using different detectors for each object category, we ensure that we detect each category as well as we can, though we allow for false positives and false negatives, which will be handled in the next stages.

2. Top-Down Pruning of Inconsistent Detections: In the second phase we use the context relations that we learned in our model to prune out nodes that support unlikely interpretations of the scene. We use a cluster-sampling algorithm (C4) (Porway and Zhu 2009) for this phase. This algorithm helps us overcome the strong coupling between objects when we sample our different interpretations of the scene. For example, Fig. 4 shows a case where we can explain a portion of the image as either a roof or a parking lot 


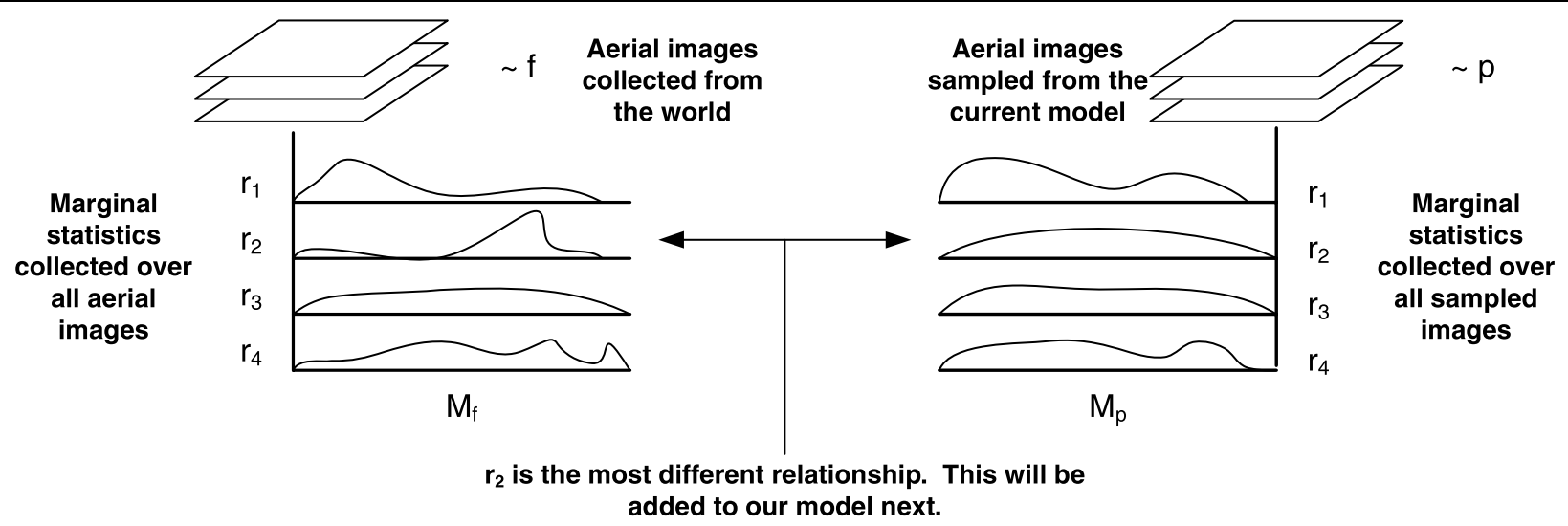

Fig. 3 A visualization of the learning process. Feature statistics $M_{f}$ and $M_{p}$ are computed over a set of aerial images and a set of aerial images sampled from our current model $p$, respectively. The most different of these, in this case $r_{2}$, is selected to be added to our model.
During the next iteration $r_{2}$ will now match between $M_{f}$ and $M_{p}$ for newly sampled images from $p$. This process continues until no feature statistics differ significantly between the two sets of images

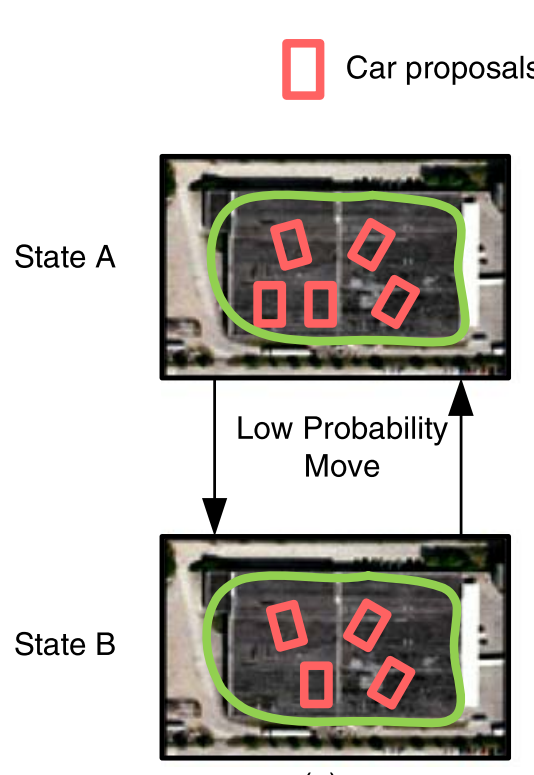

(a)
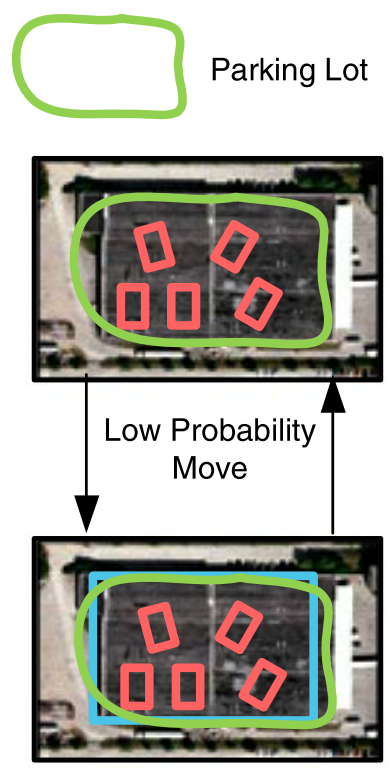

(b)

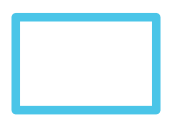

Roof

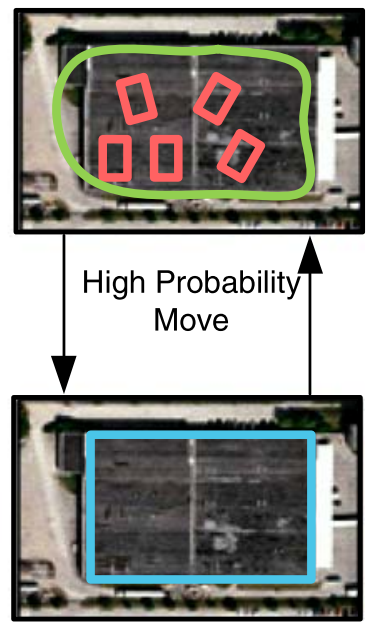

(c)
Fig. 4 The problem with dealing with strong coupling in aerial images. Here we show an image patch that can be explained either by a roof or by a parking lot with cars (often time the vents on roofs are detected as cars). Traditional sampling methods fail because the intermediate steps to get from explaining the scene as a parking lot to explaining the scene as a roof have very low probability. (a) Removing

with cars in it. If we switch from one explanation to the other by adding or removing single objects to and from our current explanation, it will take an exponentially long time to move from one explanation to the other because the intermediate steps are so unlikely.

Alternatively, adding the roof in Fig. 4(b) creates a very unlikely configuration (let's assume we never see parking lots on top of buildings in the training data), so it will be rejected. C4 clustering solves this problem by finding strongly cars one at a time just leaves an empty parking lot, which is a low probability state. (b) Adding the roof on top of the parking lot is a low probability state (we don't often see parking lots on roofs). (c) Switching the parking lot and cars for the roof in one move is a high probability move

coupled groups, like the parking lot and cars, and swapping them simultaneously with alternative explanations, as shown in Fig. 4(c).

3. Top-Down Prediction and Verification of Missing Detections: The third phase of our inference algorithm uses the top-down model to predict any missing objects based on the learned prior model and detections from Stage 2. For example, if we detected 4 cars in a row with a gap in between them, it might be reasonable to predict that another 


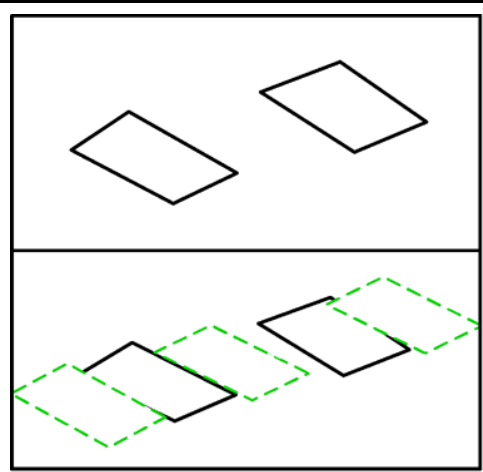

Buildings

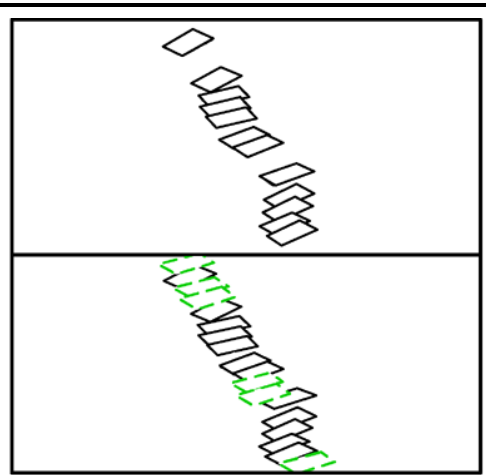

Cars

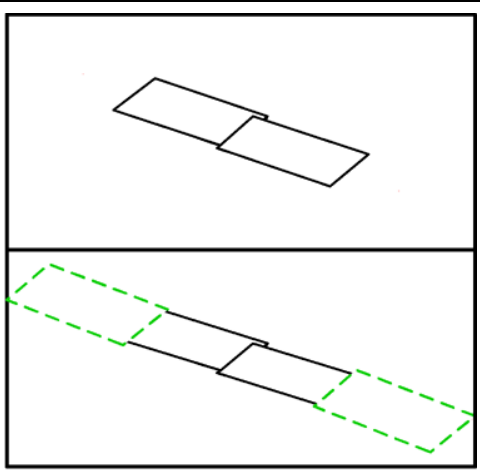

Roads

Fig. 5 (Color online) Top-down hallucinations of missing objects. Solid rectangles indicate the detections from Stage 2, while the dashed rectangles indicate hypotheses for missing objects proposed by the top-down part of our model

car should be present there. Figure 5 shows an example of predicted roofs, cars, and roads based on our results from stage two and our prior model. The hallucinated objects are shown in green dashed rectangles, while the accepted detections from $\mathrm{C} 4$ are shown in black solid rectangles. These top-down predictions will then be pruned or accepted using a final round of $\mathrm{C} 4$.

\section{Formulation}

In this section we present the probabilistic formulation for our representation from Sect. 2.1.

\subsection{Contextual Hierarchy Representation}

Our representation $\mathcal{G}$ is a 3 -tuple

$\mathcal{G}=\langle V, R, P\rangle$,

where $V$ are the nodes in the top 3 layers of Fig. 2. $R$ is a set of contextual relations and $P$ is our probability model.

The hierarchical component formed from $V$ consists of 3 types of nodes,

$V=S \cup V^{\text {Group }} \cup V^{\text {Object }}$.

\section{$S$ : The root Scene node.}

$V^{\text {Group }}$ : Groups of the same type of object, such as rows of cars or blocks of roofs.

$V^{\text {Object }}$ : Individual objects in the image.

The Scene and group nodes may decompose into one of a variable number of children nodes. This makes these nodes similar to "Or" nodes, because node $V_{i}^{\text {Group }}$ can decompose into 1 OR 2 OR 3 OR ... OR $k$ objects. We define a variable $\omega(v)$ on $v \in V$ that takes an integer value for each number of children a node $v$ decomposes into,

$\omega(v) \in\{0,1,2, \ldots, n(v)\}$.

Each node $v_{i} \in V$ has a set of attributes $\phi\left(v_{i}\right)$ that describes its position, scale, and orientation,

$\phi\left(v_{i}\right)=\left\{X_{i}, \sigma_{i}, \theta_{i}\right\}$.

In Sect. 5 we present our implementation of these attributes for our experiments.

$R=\left\{r_{1}, r_{2}, \ldots, r_{N(R)}\right\}$ is the set of relationships that exist between nodes at the same level of the hierarchy. A relationship $r_{i}$ consists of a set of $k$ nodes $V_{k} \in V$ that it acts on, a univariate function $f()$ over their attributes, and a model of the responses of $f(), p$ :

$r_{i}=\left\{V_{k}, f_{i}\left(\phi\left(V_{k}\right)\right), p_{i}\right\}$.

For example, the relative position between cars and buildings could be expressed as

$f(\phi($ Cars $), \phi($ Buildings $))=X_{\text {Cars }}-X_{\text {Buildings }}$.

If we believe relative position between cars and buildings is normally distributed with mean 5 and standard deviation 1 , then the whole relationship is packaged as

$$
\begin{aligned}
r_{i} & =\left\{(\text { Cars }, \text { Buildings }), f_{i}=X_{\text {Cars }}-X_{\text {Buildings }}, f_{i}() \sim p_{i}\right. \\
& =\mathcal{N}(5,1)\} .
\end{aligned}
$$

We will discuss the relationship functions $f()$ and their distributions $p_{i}$ in our implementation in Sect. 5. At this point it is enough to know that each relationship represents the distribution of a function response over a set of nodes. These distributions act as our statistical constraints. Figure 6 shows some examples of possible relationships. 
Fig. 6 Examples of relationships/statistical constraints. A relationship can technically be any function over the attributes of some non-empty set of nodes

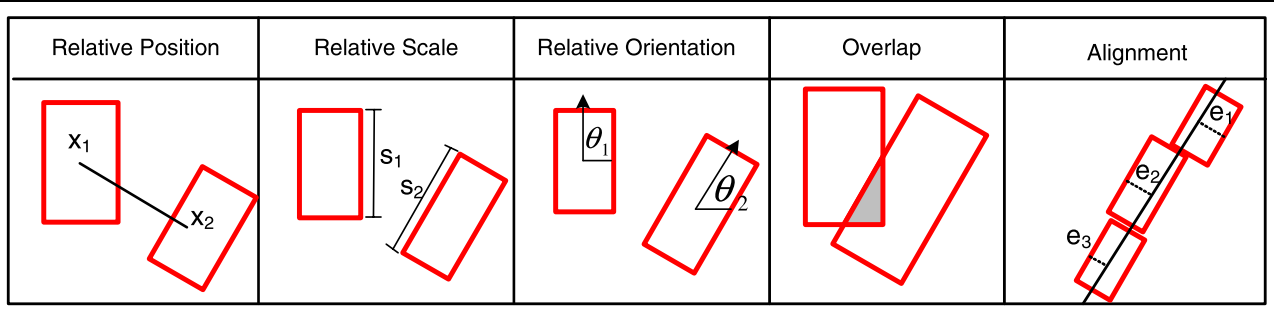

$P$ is our probability model, including the probability that nodes decompose into a certain number of child nodes, as well as the probability encoded in our statistical constraints. We will define this probability model in Sect. 3.3.

\subsection{Parse Graphs}

A parse graph, pg, is one instance drawn from the language of aerial images $\mathcal{G}$. This is like a sentence drawn from natural language and corresponds to a single aerial image. An example of a parse graph is shown in Fig. 1(c). In a parse graph the production variables $w(v)$ have been decided for every node-we've selected some number of $n$ groups from which to form the scene and selected $m_{i}$ objects to exist within each group. In addition, every group of objects in $p g$ that were constrained in $\mathcal{G}$ has inherited those appearance constraints. For example, if $\mathcal{G}$ contains a constraint on the relative distance between pairs of cars, every pair of cars in $p g \sim \mathcal{G}$ will have an edge between them constraining their relative distances.

Let us define some terminology on parse graphs, similar to that of $\mathcal{G}$ :

1. $V_{p g} \subseteq V$ : The nodes present in $p g$, which are a subset of the nodes possible in $\mathcal{G}$.

2. $\Omega_{p g}=\left\{\omega(v) ; v \in V_{p g}\right\}$ : The values of the production rules selected to form $p g$. For example, if group node $v_{i}$ consists of 6 cars, $\omega\left(v_{i}\right)=6$

3. $R_{p g} \subseteq R$ : The constraints, or edges, between nodes in $V_{p g}$. These edges are inherited from the relationships $R$ present in $\mathcal{G}$.

We should note here that parse graphs can be formed either deterministically or probabilistically. During training, we will define some grouping rules to deterministically combine labeled objects into hierarchical groups. Once labeled objects are deterministically grouped, we can measure any relationships of interest across nodes at the same level. During inference, however, the algorithm stochastically determines the most likely groupings of objects into a parse tree based on their relative appearances. The deterministic grouping function used in training is up to the user, but of course any inference methods will try to maximize the probability of a scene interpretation based on the grouping function used in learning. We describe the implementation details we use for this process in Sect. 5.2.

\subsection{Probability Model}

We begin with a set of aerial images $I^{o b s}=\left\{I_{i}^{o b s}: i=\right.$ $\left.1,2, \ldots, N^{o b s}\right\}$ that have corresponding parse graph representations $P G^{o b s}=\left\{p g_{i}^{o b s}: i=1,2, \ldots, N^{o b s}\right\}$. These parse graphs describe the hierarchy of labeled objects in the image and are deterministically constructed from labeled images as in Sect. 5.2. Each parse graph $p g$ follows some true, unknown distribution $f(p g)$. We would like the statistics of our learned model $p(p g)$ to match the statistics of $f(p g)$ as closely as possible. The statistics of $f(p g)$ consist of

1. The distribution of $\omega\left(v_{(\alpha)}\right)$, the number of children each node $v_{(\alpha)} \in V$ decomposes into.

2. The distribution of responses of $f_{(\beta)}()$ for each statistical relation $r_{(\beta)} \in R$ in $\mathcal{G}$.

Note that we are switching our indexing subscripts from $i$ 's to $\alpha$ 's and $\beta$ 's for clarity. $\alpha$ subscripts will be used when we are referring to the distributions of node decompositions, and $\beta$ subscripts will be used when we are referring to the distributions of relationship constraints.

We will model both node decomposition and relationship distributions as histograms for the remainder of the paper. Specific parametric models may fit the distributions of $\omega\left(v_{(\alpha)}\right)$ and $f_{(\beta)}()$ more closely, but we use histograms so that we can focus the discussion on learning the model without additional parameters. Also, histograms measure the true continuous distributions of $f(p g)$ and $p(p g)$ in the limit. We approximate the continuous distribution by the piecewise-continuous representation of histograms for each node $v_{(\alpha)} \in V$ and each $f_{(\beta)} \in R$. Our observed statistics for each bin $z$ of the histograms for $\omega\left(v_{(\alpha)}\right)$ and $f_{(\beta)}()$ are then

$$
\begin{gathered}
H_{(\alpha)}(p g, z)=\frac{\sum_{i=1}^{N^{o b s}} \#\left(\omega\left(v_{(\alpha)}\right)=z\right)}{\sum_{i=1}^{N^{o b s}} \sum_{j=0}^{n\left(v_{(\alpha)}\right)} \#\left(\omega\left(v_{(\alpha)}\right)=j\right)}, \\
\alpha=1,2, \ldots, n(V) \\
H_{(\beta)}(p g, z)=\sum_{i=1}^{N^{o b s}} \frac{\sum_{V_{(\beta)} \subseteq V_{p g(i)}} \#\left(f_{(\beta)}()=z\right)}{\sum_{V_{(\beta)} \subseteq V_{p g(i)}} \#\left(f_{(\beta)}()\right)}, \\
\beta=1,2, \ldots, n(R)
\end{gathered}
$$

where \# is a counting function representing the number of times that something occurs, and $\#\left(f_{(\beta)}()\right)$ is the number of 
times $f_{(\beta)}$ takes any value. Each bin $z$ in $H_{(\alpha)}(p g)$ is the number of times that node $v_{(\alpha)}$ decomposes into $z$ children divided by the number of times we observe $v_{(\alpha)}$ decomposing any of its $n\left(v_{(\alpha)}\right)$ values. Each bin $z$ in $H_{(\beta)}(p g)$ is the number of times that relationship function $f_{(\beta)}$ returns $z$ divided by the number of times $f_{(\beta)}$ returns anything.

We seek a distribution $p(p g)$ that matches the relationship statistics $\left(H_{(\alpha)}(p g), H_{(\beta)}(p g)\right)$ as closely as possible with $f(p g)$, while remaining as random as possible (unprejudiced) along all other dimensions. This is equivalent to making sure that the expectation of the number of objects in each group, $E_{p}\left[\omega\left(v_{(\alpha)}\right)\right]$, and the expectation of each relationship function, $E_{p}\left[f_{(\beta)}\left(\phi\left(V_{(\beta)}\right)\right)\right]$, matches between our model and the true distribution. By maximum entropy this becomes the following constraint satisfaction problem,

$p(p g)^{*}=\arg \max \left\{-\sum p(p g) \log p(p g)\right\}$

subject to

$$
\begin{aligned}
& \quad E_{p}\left[\omega\left(v_{(\alpha)}\right)\right]=E_{f}\left[\omega\left(v_{(\alpha)}\right)\right], \quad \alpha=1,2, \ldots, n(V) \\
& E_{p}\left[f_{(\beta)}\left(\phi\left(V_{(\beta)}\right)\right)\right]=E_{f}\left[f_{(\beta)}\left(\phi\left(V_{(\beta)}\right)\right)\right], \\
& \quad \beta=1,2, \ldots, n(R) \\
& E_{f}\left[\omega\left(v_{(\alpha)}\right] \approx H_{(\alpha)}\left(P G^{o b s}\right)\right. \\
& E_{f}\left[f_{(\beta)}\left(\phi\left(V_{(\beta)}\right)\right)\right] \approx H_{(\beta)}\left(P G^{o b s}\right)
\end{aligned}
$$

In other words, we want the histograms formed from sampled aerial images from our model to match the true distributions observed in the training data. The probability model that satisfies these constraints is the familiar Gibbs model

$$
\begin{aligned}
& p(p g ; \Theta, R)=\frac{1}{Z[\Theta]} \exp ^{-\xi(p g)} \\
& \xi(p g)=\sum_{\alpha=1}^{n(V)}\left\langle\lambda_{(\alpha)}, H_{(\alpha)}(p g)\right\rangle+\sum_{\beta=1}^{n(R)}\left\langle\lambda_{(\beta)}, H_{(\beta)}(p g)\right\rangle \\
& Z[\Theta]=\sum_{p g \in L(\mathcal{G})} \exp ^{-\xi(p g)}
\end{aligned}
$$

where $\Theta=\left\{\lambda_{(\alpha)}, \lambda_{(\beta)}\right\}$. The first term in $\xi(p g)$ is the energy of the decomposition rules and the second is the energy of the relationship constraints. If we have an unlikely number of objects in an image (say 0 objects), then the first term will have high energy and the interpretation will have low probability. If we have objects that do not obey the statistical constraints we learned during training, for example we observe a car on top of a tree, then the second term will have high energy and the interpretation will have low probability.

The Lagrange multipliers $\left\{\lambda_{(\alpha)}, \lambda_{(\beta)}\right\}$ are vectors of the same dimension as $H_{(\alpha)}$ or $H_{(\beta)}$, respectively, and $\langle\ldots\rangle$ indicates an inner product. For example, if relationship $r_{(\beta)}$ 's function $f_{(\beta)}$ evaluates to $z$ on parse graph $p g$, then the energy from that relationship is $\lambda_{(\beta)}^{z} * H_{(\beta)}(p g, z)$. The $\lambda$ 's are the natural parameter set of the model and serve to weight histogram bins so that dependent relationship interactions are weighted correctly. These $\lambda$ 's will be learned in the following section.

\section{Learning the Hierarchical Contextual Model}

We begin with a set of $N^{o b s}$ aerial images $I^{o b s}=\left\{I_{i}^{o b s}\right.$ : $\left.i=1,2, \ldots, N^{o b s}\right\}$ and their corresponding parse graphs $P G^{o b s}=\left\{p g_{i}^{o b s}: i=1,2, \ldots, N^{o b s}\right\}$. The parse graphs $P G^{o b s}$ follow the real-world, unknown target distribution, $f(p g)$, by definition,

$p g^{o b s} \sim f(p g)$.

Matching our distribution $p(p g)$ to $f(p g)$ is equivalent to finding the values for $\Theta$ that minimize the KL divergence between the two distributions

$$
\begin{aligned}
\Theta^{*} & =\arg \min _{\Theta} K L(f(p g) \| p(p g ; \Theta, R)) \\
& =\arg \min _{\Theta} \sum_{p g} f(p g) \log \frac{f(p g)}{p(p g ; \Theta, R)},
\end{aligned}
$$

which is equivalent to finding the maximum likelihood estimates for $\Theta$ and a set of relationships $R$ constraining the model. Letting $\mathcal{L}(\Theta)$ be the log-likelihood function for our parameters,

$$
\begin{aligned}
& \mathcal{L}(\Theta)=\sum_{p g \in P G^{\text {obs }}} \log p(p g ; \Theta, R) \\
& (\Theta, R)^{*}=\underset{(\Theta, R)}{\arg \max _{(} \mathcal{L}(\Theta) .}
\end{aligned}
$$

Learning our parameters can then be broken down into two distinct stages:

1. Given a set of relationships $R$ in the model, estimate $\left(\lambda_{(\alpha)}, \lambda_{(\beta)}\right)$.

2. Pursue a set of relationships $R$ one-by-one to constrain the model.

This may seem backwards, but it is easier to understand the process if we first show the process for parameter estimation given a set of relationships $R$ followed by the process for pursuing $R$.

\subsection{Learning $\left(\lambda_{(\alpha)}, \lambda_{(\beta)}\right)$}

We solve for $\Theta=\left(\lambda_{(\alpha)}, \lambda_{(\beta)}\right)$ using straightforward maximum likelihood estimation (MLE). Setting $\frac{\partial \mathcal{L}}{\partial \Theta}=0$, we can solve for both sets of $\lambda$ 's: 
1. $\lambda_{(\alpha)}$ : We make the assumption that node decompositions are independent of each other and of their appearance constraints. We can therefore model $H_{(\alpha)}(p g)$ as a multinomial and count the frequency with which each node decomposes into a number of children, as in (10). This makes our estimate for each $\lambda_{(\alpha)}$

$\lambda_{(\alpha)}=-\log H_{(\alpha)}\left(P G^{o b s}\right)$.

This is the MLE estimate for a multinomial and can be used to estimate production rule probabilities in grammars given that they are independent of any cross-link relations, i.e. context-free (Chi and Geman 1998).

2. $\lambda_{(\beta)}$ : Setting $\frac{\partial \mathcal{L}}{\partial \Theta}=0$ for $\lambda_{(\beta)}$ yields

$E_{p}\left[f_{(\beta)}\left(\phi\left(V_{(\beta)}\right)\right)\right]=H_{(\beta)}\left(P G^{o b s}\right)$

which are exactly the Lagrange constraints that resulted from deriving our maximum entropy model. This equation can also be written as

$\frac{\partial \mathcal{L}}{\partial \lambda_{(\beta)}}=\frac{1}{N^{o b s}} \sum_{p g \in P G^{o b s}} H_{(\beta)}(p g)-E_{p}\left[H_{(\beta)}\left(P G^{o b s}\right)\right]$

which can be approximated by

$\frac{\partial \mathcal{L}}{\partial \lambda_{(\beta)}} \approx H_{(\beta)}\left(P G^{s y n}\right)-H_{(\beta)}\left(P G^{o b s}\right)$.

$H_{(\beta)}\left(P G^{s y n}\right)$ is the histogram formed from a set of parse graphs $P G^{s y n}=\left\{p g_{i}^{s y n}: i=1,2, \ldots, N^{s y n}\right\}$ that are synthesized from our current model $p(p g)$. The synthesized parse graphs are drawn by first sampling the number of children each node decomposes into according to the learned $\lambda_{(\alpha)}$ parameters. The appearances of the objects in the resulting parse tree are then Gibbs sampled according to the current $\lambda_{(\beta)}$ weights and the constraints in the model. These images will also be aerial images, so we can compute histograms for the same relationship function $f_{(\beta)}()$ over these parse graphs as we did over $P G^{o b s}$.

Solving for the $\lambda_{(\beta)}$ 's such that $H_{(\beta)}\left(P G^{\text {syn }}\right)=$ $H_{(\beta)}\left(P G^{o b s}\right)$ can then be done using gradient descent. We initialize the $\lambda_{(\beta)}$ weights to 0

$\lambda_{(\beta)}=0, \quad \beta=1,2, \ldots, n(R)$.

In the first stage when $\lambda_{(\beta)}=0, H_{(\beta)}\left(P G^{s y n}\right)$ will be close to uniform. Gradient descent is then used to update the $\lambda_{(\beta)}$ 's,

$\lambda_{(\beta)}^{t+1}=\lambda_{(\beta)}^{t}-\eta\left(H_{(\beta)}\left(P G^{s y n}\right)-H_{(\beta)}\left(P G^{o b s}\right)\right)$,

where $\eta$ is a step factor that can depend on the iteration $t$. This update reweights the $\lambda_{(\beta)}$ 's for each histogram based on how much $H_{(\beta)}\left(P G^{s y n}\right)$ differs from $H_{(\beta)}\left(P G^{o b s}\right)$. It reduces the energy for choosing underrepresented bins and increases the energy for choosing overrepresented bins during the next iteration of Gibbs sampling. After a number of iterations the $\lambda_{(\beta)}$ 's will be weighted such that the synthesized distributions match the observed distributions, and thus $p(p g)$ will match $f(p g)$ along these dimensions. In other words,

$\left|H_{(\beta)}\left(P G^{s y n}\right)-H_{(\beta)}\left(P G^{o b s}\right)\right|<\varepsilon_{2}, \quad \beta=1,2, \ldots, n(R)$.

Figure 7 shows a toy example of the $\lambda_{(\beta)}$ learning process. We begin with one observed histogram $H^{\text {obs }}$ for relative car size and its corresponding $\lambda$ weight vector, which begins as a vector of all 0's. Because this weight is uniform, the images we sample in Step (2) look fairly random. In Step (3) we compute $H^{s y n}$, the distribution of relative car sizes across these sampled images. This, unsurprisingly, is fairly uniform as well because $\lambda$ was uniform. The figure shows these $H^{s y n}$ and $H^{o b s}$ superimposed below to emphasize the difference in their bin counts. In Step (4) we
Fig. 7 Examples of learning the relationship parameters, $\lambda_{(\beta)}$. (1) We begin with an observed histogram $H^{o b s}$, in this case the relative size between cars. $\lambda$ begins uniform. (2) Sampled images $P G^{\text {syn }}$ are drawn from the model. (3) $H^{s y n}$ is computed for relative car size over the sampled images. (4) $\lambda$ is reweighted according to the difference between $H^{o b s}$ and $H^{s y n}$. (5) Newly sampled images appear scaled correctly

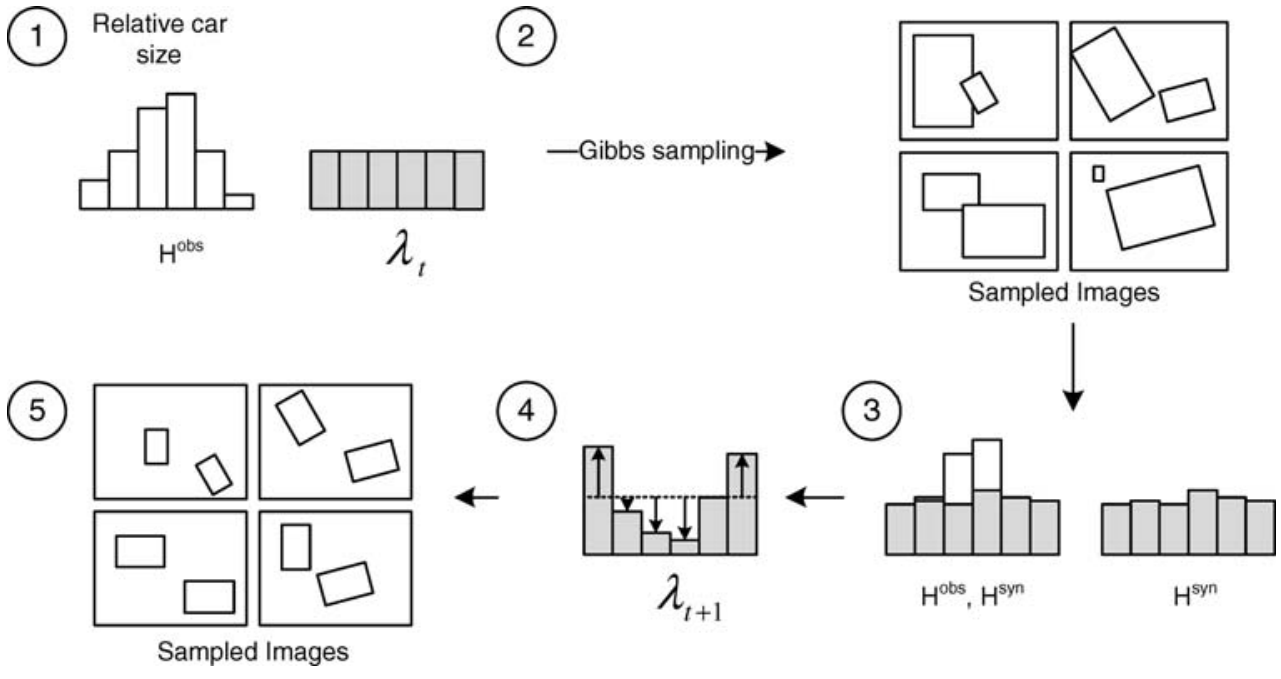


update the $\lambda$ weights according to how much each bin differs. Step (5) shows the sampled images resulting from the updated $\lambda$ weighting, which have much more appropriate relative car sizes. This process is carried out simultaneously for each $\lambda_{(\beta)}$.

\subsection{Relationship Pursuit}

The $\lambda_{(\beta)}$ 's above were learned given that we already knew which relationships $R$ existed in the model. We now show how to select the relationship constraints for the model. Because our dictionary of potential relationships $\Delta_{R}$ could be combinatorially huge, we will iteratively add relationships according to their importance instead of fitting the full model. Fitting the full model would require later attempts to sample from the model or perform inference with the model to check redundant relationships, making the model overly complex and slower to compute with.

We pursue the relationship set $R$ by beginning with just an empty hierarchy,

$p_{0}\left(p g ; \Theta_{0}, R_{0}\right) ; \quad R_{0}=\{\emptyset\}$.

This is the model with no parameters learned at all. We then learn the $\lambda_{(\alpha)}$ 's, or tree parameters, allowing us to sample images with the correct distributions of objects, but without spatial or appearance constraints. Sampling the model at this stage would produce parse graphs with the correct number of objects, but without horizontal constraints, causing the resulting image to look more like an "alphabet soup" of objects that are big, small, overlapping, etc. We then iteratively add a new relationship $r_{+}$from a dictionary of potential relationships $\Delta_{R}$ at each iteration to get a new distribution $p_{+}\left(p g ; \Theta_{+}, R_{+}\right), R_{+}=R \cup\left\{r_{+}\right\}$. We choose $r_{+}$such that we minimize $K L\left(f(p g) \| p_{+}\left(p g ; \Theta_{+}, R_{+}\right)\right)$at each step:

$$
\begin{aligned}
p_{0}\left(p g ; \Theta_{0}, R_{0}\right) & \rightarrow p_{1}\left(p g ; \Theta_{1}, R_{1}\right) \rightarrow \cdots \\
& \rightarrow p_{k}\left(p g ; \Theta_{k}, R_{k}\right) .
\end{aligned}
$$

At each iteration we want to select the relation $r_{+}$that brings our new model $p_{+}$closest to $f$, thereby reducing the KL divergence between the two distributions. This is equivalent to finding the new $p_{+}$that is maximally KL divergent from our current $p$, as visualized in Fig. 3. Because we are guaranteed to monotonically decrease $K L\left(f(p g) \| p_{+}\left(p g ; \Theta, R_{+}\right)\right)$with every added relation (Zhu et al. 1998), the $r_{+}$that is maximally far away from $p$ must be maximally close to $f$ and thus represents the largest decrease in KL divergence:

$$
\begin{aligned}
r_{+}= & \underset{r}{\operatorname{argmax}} K L(f(p g) \| p(p g ; \Theta, R)) \\
& -K L\left(f(p g) \| p_{+}\left(p g ; \Theta_{+}, R_{+}\right)\right) \\
= & \underset{r}{\operatorname{argmax}} K L\left(p_{+}\left(p g ; \Theta_{+}, R_{+}\right) \| p(p g ; \Theta, R)\right) .
\end{aligned}
$$

We can approximate this decrease in KL divergence, otherwise knows as the information gain, $\delta\left(r_{+}\right)$, using the Mahalanobis distance between the synthesized and observed histograms for the new potential relation $r_{+}$

$$
\begin{aligned}
\delta\left(r_{+}\right) & =K L\left(p_{+}\left(p g ; \Theta_{+}, R_{+}\right) \| p(p g ; \Theta, R)\right) \\
& \approx d_{m a h n}\left(H_{(\beta)}^{r_{+}}\left(P G^{o b s}\right), H_{(\beta)}^{r_{+}}\left(P G^{s y n}\right)\right) .
\end{aligned}
$$

This holds due to a Taylor expansion around the relationship we're interested in adding, as shown in the Appendix of Zhu et al. (1998). We measure $H_{(\beta)}\left(P G^{o b s}\right)$ and $H_{(\beta)}\left(P G^{s y n}\right)$ for all relations $r_{(\beta)} \in \Delta_{R}$ and compare their Mahalanobis distances. The $r_{(\beta)}$ with the largest Mahalanobis between the synthesized and observed histograms above some threshold is added to the model in the next iteration and its $\lambda_{(\beta)}$ parameters are learned as in the previous section.

\subsection{Summary of Parameter Learning and Relationship Pursuit Algorithms}

The algorithm for learning the parameters of the model proceeds in two steps. We first learn the $\lambda_{(\alpha)}$ 's by MLE, which are just the sample frequencies of the decompositions of each node. We then iteratively add spatial and appearance relations one-by-one until no relation remaining in $\Delta_{R}$ has Mahalanobis distance greater than $\varepsilon_{1}$. After each relation is added, we iteratively update the $\lambda_{(\beta)}$ 's for the current relation set to match $H_{(\beta)}\left(P G^{s y n}\right)$ to $H_{(\beta)}\left(P G^{o b s}\right), \forall r_{(\beta)} \in R$. The algorithms are outlined below:

\section{Algorithm 1 Relationship pursuit}

1. Begin with an empty model $p_{0}$ and observed parse graphs $P G^{o b s}=\left\{p g_{i}^{o b s}: i=1,2, \ldots, N^{o b s}\right\}$.

2. Compute observed histograms $H_{(\beta)}\left(P G^{o b s}\right)$ and $H_{(\alpha)}\left(P G^{o b s}\right)$ for all relationships in $\Delta_{R}$ and all node frequencies, respectively.

3. Approximate the $\lambda_{(\alpha)}$ 's for the tree component using MLE, yielding $p_{t}$.

4. Repeat

(a) Sample $N^{\text {syn }}$ parse graphs from the current model, $P G^{\text {syn }}=\left\{p g_{i}^{\text {syn }}: i=1,2, \ldots, N^{s y n}\right\}$.

(b) Calculate $H_{(\beta)}\left(P G^{s y n}\right), \beta=1,2, \ldots,\left|\Delta_{R}\right|$.

(c) Select the $r_{(\beta)}$ for which $d_{\text {manh }}\left(H_{(\beta)}\left(P G^{s y n}\right), H_{(\beta)}\left(P G^{o b s}\right)\right)$ is maximal as $r_{+}$. Add $r_{+}$to $R$.

(d) Relearn the $\lambda_{(\beta)}$ 's for the new set $R \cup\left\{r_{+}\right\}$using Algorithm 2.

until $d_{\operatorname{manh}}\left(H_{(\beta)}\left(P G^{s y n}\right), H_{(\beta)}\left(P G^{o b s}\right)\right)<\varepsilon_{1}, \beta=$ $1,2, \ldots,\left|\Delta_{R}\right|$. 
Algorithm 2 Parameter estimation algorithm

1. Given a set of relations $R$ and current model $p(p g ; \Theta, R)$,

2. Repeat

(a) Sample $n$ parse trees from the model, $P G^{s y n}=$ $\left\{p g_{i}^{s y n}: i=1,2, \ldots, N^{s y n}\right\}$.

(b) Calculate $H_{(\beta)}\left(P G^{s y n}\right), \beta=1,2, \ldots, n(R)$.

(c) Update $\lambda_{(\beta)}^{t+1}=\lambda_{(\beta)}^{t}-\left(\eta\left(H_{(\beta)}\left(P G^{s y n}\right)-\right.\right.$ $\left.\left.H_{(\beta)}\left(P G^{o b s}\right)\right)\right)$.

until $\left|H_{(\beta)}\left(P G^{s y n}\right)-H_{(\beta)}\left(P G^{o b s}\right)\right|<\varepsilon_{2}, \beta=$ $1,2, \ldots, n(R)$.

\section{Implementation}

The learning algorithm above is independent of our choice of models for the relationship statistics and the object representations. In this section we present implementation details used to learn the model above in our experiments.

\subsection{Object Representation}

We represent our five object categories, (roads, trees, roofs, cars, parking lots), by their enclosing boundaries. Each object of interest is described by a boundary $b$ that is defined as a graph,

$b_{i}=\left\langle v_{i}, \zeta_{i}\right\rangle$

where $v$ is a set of boundary points and $\zeta$ is a set of edges, along with a label $l_{i}$ indicating what type of object it is. The boundaries and labels are hand-labeled in every observed image $I^{o b s}$. These objects form the bottom layer of the hierarchy. We create a node $v_{i}$ in the 3-layer hierarchy for each boundary $b_{i}$ so that every boundary is represented by a bottom-level node in the hierarchy and every bottom-level node in the hierarchy has a corresponding boundary representation.

From these boundaries we can derive the appearance attributes $\phi\left(v_{i}\right)=\phi\left(b_{i}\right)=\left\{X_{i}, \theta_{i}, \sigma_{i}\right\}$ of each boundary $b_{i}$ for each bottom-level node $v_{i}$ in the hierarchy. The smallest enclosing bounding box, box $_{i}$, was computed for each $b_{i}$. The position of each object is its center of mass, its orientation is the major axis of $b o x_{i}$, and its scale is the length and width of $b o x_{i}$, treating the major axis as our measure of length and the minor axis our measure of width. If boundary $b_{i}$ for node $v_{i}$ consists of $n$ vertices $v=\left\{v_{1}, v_{2}, \ldots, v_{n}\right\}$ and $\left(\right.$ left $_{i}$, right $_{i}$, top $_{i}$, bottom $_{i}$ ) describe the center points of the edges of $b_{i}$ 's bounding box box $x_{i}$, we can compute each object's appearance attributes as,

$X_{i}=\left(\frac{\sum_{j=1}^{n} x\left(v_{j}\right)}{n}, \frac{\sum_{j=1}^{n} y\left(v_{j}\right)}{n}\right)$

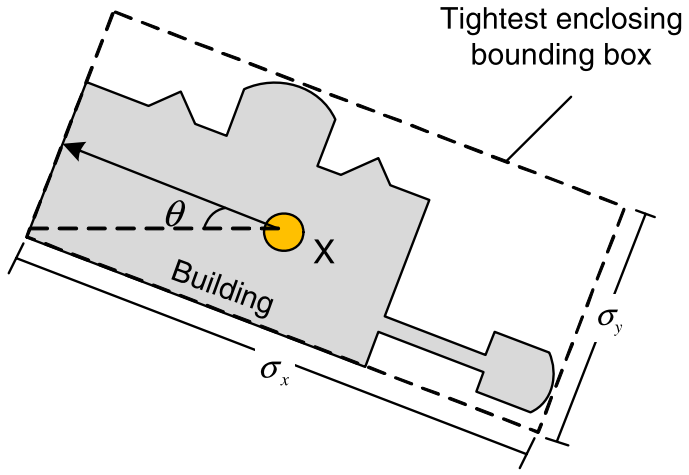

Fig. 8 An example of the features computed for a single object. The boundary graph and smallest enclosing bounding box are used to compute position, scale, and orientation

$$
\begin{aligned}
& M_{\text {axis }}=\max \left(\left(\text { right }_{i}-\text { left }_{i}\right),\left(\text { top }_{i}-\text { bottom }_{i}\right)\right) \\
& m_{\text {axis }}=\min \left(\left(\text { right }_{i}-\text { left }_{i}\right),\left(\text { top }_{i}-\text { bottom }_{i}\right)\right) \\
& \theta=\cos ^{-1}\left(M_{\text {axis }},(1,0)\right) \\
& \sigma=\left(\left|M_{\text {axis }}\right|,\left|m_{\text {axis }}\right|\right) .
\end{aligned}
$$

Figure 8 shows an example of the representation of a building based on these features.

\subsection{Deterministically Forming Parse Graphs}

Each $I^{o b s}$ has a corresponding parse graph $\mathrm{pg}^{\text {obs }}$ describing the hierarchical arrangement of its objects. We are given the boundaries of every object as described in Sect. 5.1. We touched briefly on the deterministic formation of parse graphs in Sect. 3.2, but now go into more details of our implementation for deterministically converting labeled objects into parse graphs.

To form parse graphs from a collection of labeled objects (the leaf nodes) we make the following stipulation:

Proposition 1 Boundaries within distance $\varrho$ of each other that are of the same object label will be considered members of the same group.

In other words, objects are deterministically assigned to groups according to their distance between one another. This provides two benefits:

(1) We can deterministically form a hierarchy from a flat set of objects.

(2) We only measure relationship statistics within and between groups of objects, so limiting the distance at which two objects are related prevents us from calculating and learning statistics of objects that are very far away.

In our experiments we set $\varrho$ to be label-dependent. If we let $s$ be $b_{i}$ 's aspect ratio, we can define a set of distance thresholds as in Table 1. For example, a tree would 
need to be within 1.2 of its aspect ratio of another tree to be considered in the same group. Because some objects within the same label may vary significantly in size, one may also choose to consider two objects proximal only if they are within $c * \min \left(s_{1}, s_{2}\right)$ of each other, where $\left(s_{1}, s_{2}\right)$ are the aspect ratios of the two objects in question. This is particularly useful when determining which groups of objects should be associated, as their sizes can vary significantly more than those of single objects.

Figure 9 shows an example of deterministically forming a parse graph from a set of labeled objects. In this example, cars that are nearby one another are grouped together, as shown in (a). The same goes for roofs labeled in (a). Figure 9(b) shows the resulting groups from this first step and their distance-based relationships as well.

Table 1 Category-dependent distance thresholds $\varrho$ for deterministic grouping based on an object's aspect ratio $s$

\begin{tabular}{ll}
\hline Object label & $\varrho$ \\
\hline Roof(s) & $s$ \\
Car(s) & $0.5 * s$ \\
Tree(s) & $1.2 * s$ \\
Road(s) & $0.5 * s$ \\
Parking Lot(s) & $s$ \\
\hline
\end{tabular}

An important point to note here is that, though we form parse graphs deterministically for our observed images, we do not form them deterministically when inferring the best explanation of a new image. In our training images $I^{\text {obs }}$ we are making the assumption that proximal objects are grouped and that this grouping defines the number of objects that the group consists of (decomposes into). There may, however, be proximal groups in our testing images that have, for example, a different number of objects than we expect to see based on our training data. In this case, it may make more sense to split the group into subgroups that match our learned decomposition frequencies than grouping them all under a hard-coded proximity condition. In a world where we've only seen sets of three cars, a row of six cars is more consistently explained as two sets of three by our model.

\subsection{Relationship Functions}

The functions $f_{i}$ for each relationship are defined over the attributes of sets of nodes, $\phi\left(V_{i}\right)$. We implemented the relationship functions listed in Table 2. The relationship functions should be fairly explanatory, with the exception of the position functions. Relative position returns the vector between the centers of the two objects, which is relative to the coordinate frame of the image. This is not particularly useful as aerial images rarely have a well-defined "top"
Fig. 9 An example of deterministically forming the neighborhood structure for a parse graph from labeled objects. (a) Cars and roofs that are within a certain distance of each other are grouped together. (b) Groups that are within a certain distance of each other have group-level constraints applied

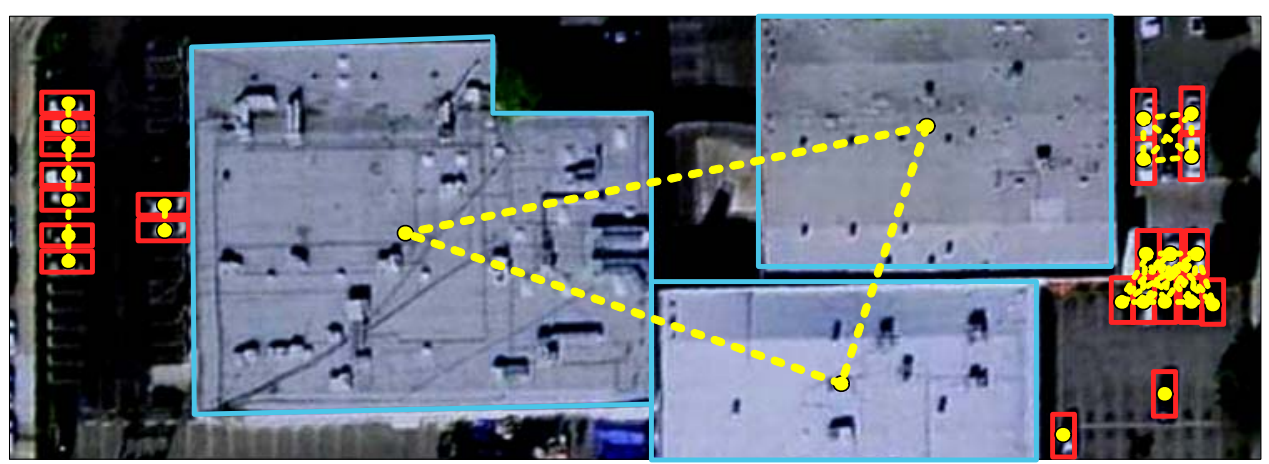

(a) Network structure between single objects

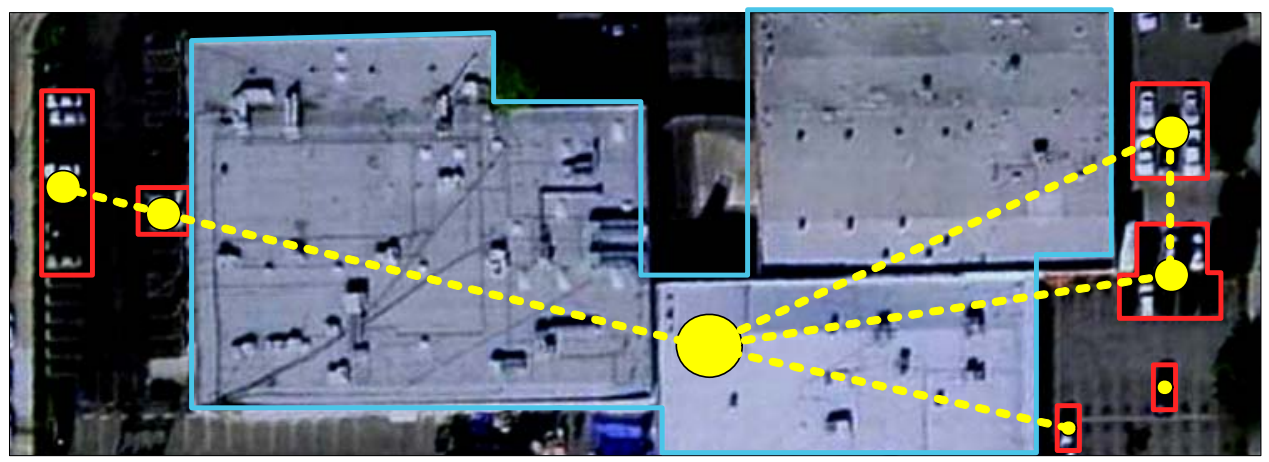

(b) Network structure between grouped objects

Object/Group boundary

Object/Group center Neighborhood Edge 
Table 2 Relationship function definitions

\begin{tabular}{lll}
\hline Relationship & $n$ Nodes & Function $f_{i}()$ \\
\hline Aspect ratio & 1 & $\sigma_{y} / \sigma_{x}$ \\
Relative position (in image coordinate frame) & 2 & $\left(X_{2}-X_{1}\right) / s_{1}$ \\
Radial position (in object coordinate frame) & 2 & $\left\{\left|X_{2}-X_{1}\right|, \theta\left(\left|X_{2}-X_{1}\right|\right)-\theta_{1}\right\}$ \\
Relative scale & 2 & $s_{1} / s_{2}$ \\
Relative orientation & 2 & $\theta_{2}-\theta_{1}$ \\
Percentage overlap & 2 & Area \\
Alignmerlap $/$ Area $_{1}$ \\
\hline
\end{tabular}

or "bottom". Radial position attempts to deal with this by measuring relative distance in polar coordinates. These twodimensional features can also be decoupled and collected as if they were independent, e.g. a relationship of just relative $\mathrm{X}$ or $\mathrm{Y}$ positions or of distance and relative angle. The responses of these functions form the histograms $H_{(\beta)}$ that we match in the learning stage.

\subsection{Histograms}

We have chosen to represent our relationship statistic distributions as histograms. This is intended to save us the trouble of fitting specific distributions to each new relationship. In order to use histograms, we made the following design decisions:

1. The range of the histograms are determined by the maximum and minimum values observed in the training data. We model values outside of this range by a decreasing gradient function. Define the maximum probability value allowed for this gradient function as $p$ and the width of the histogram bin as $w$. Then a point that is distance $k$ bin widths beyond the edge of the histogram (i.e. $k * w$ distance from the edge) can be assigned probability $p-(k / n) * p$, where $n$ is as many extra bins as we'd like to add to either side of our histogram. Thus, values that are beyond the histogram edge are assigned a probability that is a fraction of the maximum probability $p$, depending on how far away they are. $n$ is usually set to be something large, e.g. 10000 , and $p$ is usually set to be the edge bin probability, which prevents the tail from being greater probability than the probability in the edgemost bins. Values greater than $n$ are assigned probability 0 (or some minimum probability). These tails allow us to model values outside of the histogram range while guaranteeing that their probability is never greater than the probability for the edge closest to that side.

2. We divide our histograms into 10 bins each. We were surprised to find that, empirically, any number of bins above 6-7 were sufficient to produce samples from the model that are perceptually similar to real aerial images. Obviously we will never perfectly match the distributions with this discretization until we approach the limit, but we find suitable results with even as few as 10 bins.

3. Our training set is intentionally small, so that little human intervention is needed. At first it may appear that we are therefore reducing the size of our training set and will not have sufficient data to model the distributions. However, as most of our images are large images containing hundreds of the objects, one image often provides a large number of data points.

\section{Experiments on Learning and Sampling}

We selected 120 aerial images from the Lotus Hill Database (Yao et al. 2007), which included labeled boundaries of roofs, roads, parking lots, tree regions, and cars to use as our training data. ${ }^{1}$ As mentioned in Sect. 1, these boundaries are hand-labeled. The images ranged in size from $640 \times 480$ pixels to $1000 \times 1000$ pixels. We set $\varepsilon_{1}=4$ and $\varepsilon_{2}=0.2$ for Algorithms 1 and 2 and then learned a hierarchical contextual model of objects in aerial images.

Figure 10 shows $H_{(\beta)}\left(P G^{o b s}\right)$ and $H_{(\beta)}\left(P G^{s y n}\right)$ for four typical relations at three iterations of the parameter learning algorithm. In the first iteration, the histograms from our synthesized images are so far away from the true histograms that most of their data is out of bounds. Halfway through the learning, however, the histograms start to look coarsely similar. By the final iteration, the histograms have matched nearly perfectly. This assures us that the $\lambda_{(\beta)}$ 's are reweighting the histogram bins correctly such that, over time, the images we synthesize using our model match the statistics of true aerial images.

We used 5 object categories in our model (car, roof, road, parking lot, tree) and their 5 corresponding group categories. We used 7 relationship functions in our model, resulting in a relationship dictionary $\Delta_{R}$ consisted of 360 possible relationships (10 aspect ratio relations $+(5$ objects $) *$ $(5$ objects $) *(7$ relationships $)+(5$ groups $) *(5$ groups $) *$

\footnotetext{
${ }^{1}$ Dataset available from http://www.imageparsing.com. More data will be released after the publication of this paper, but sample data is available free for downloading now.
} 


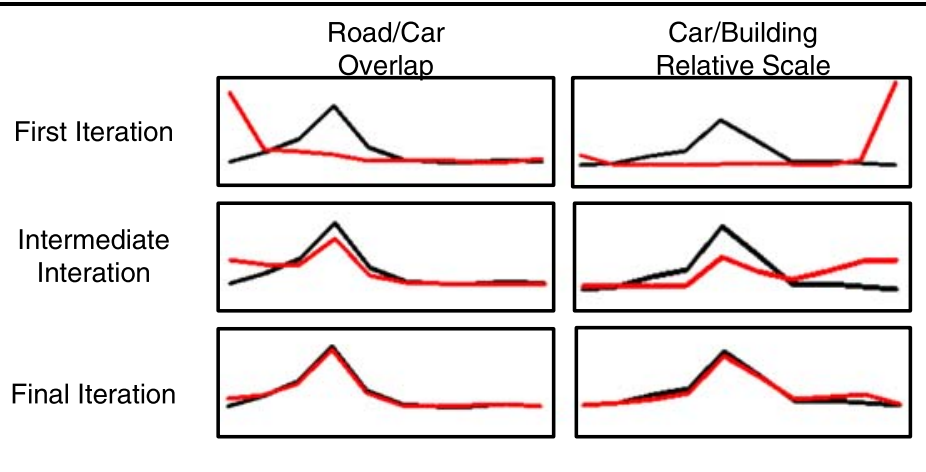

Observed histograms

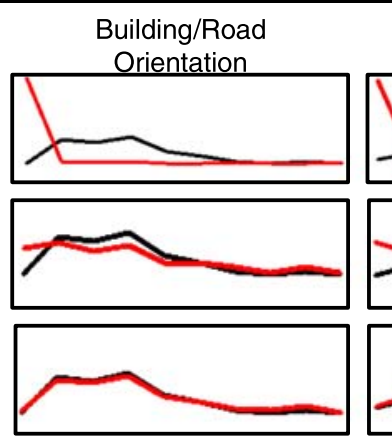

Parking Lot/Car Overlap

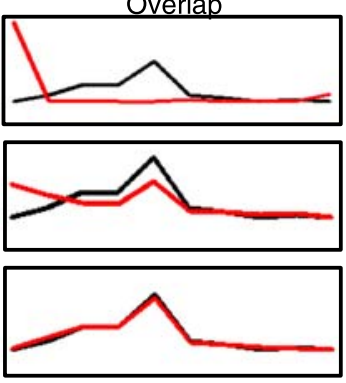

Synthesized histograms
Fig. 10 (Color online) Histograms for four typical relations over the course of the learning algorithm. The black lines are the histograms of the observed data, $H_{(\beta)}\left(P G^{o b s}\right)$, and the red lines are the histograms of the synthesized data, $H_{(\beta)}\left(P G^{s y n}\right)$, at each iteration. At first the statis- tics of the synthesized data are so far off from the truth that most values are out of bounds. Halfway through the learning process the histograms look close to matching and by the final iteration the histograms match nearly perfectly
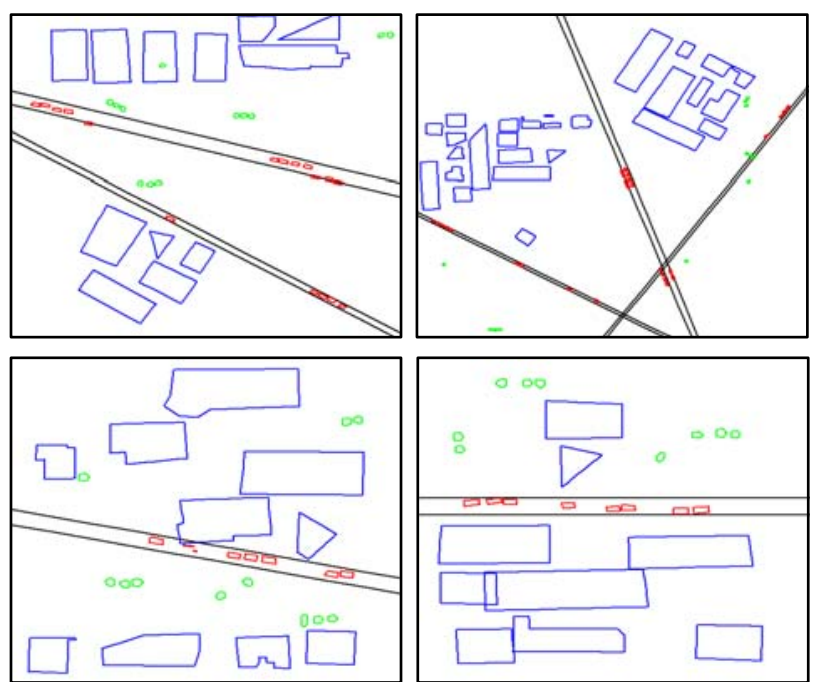

Fig. 11 (Color online) Samples from our learned model (blue = roofs, red $=$ cars, black $=$ roads, green $=$ trees). These are not images directly sampled from the training data, but collections of objects obey-

(7 relationships)). Of those 360 possible relationships our model selected 27 , consisting mostly of overlap relations (car/car overlap, building/tree overlap, car/parking lot overlap), relative scale relations (car/car relative scale, roof/road relative scale), and alignment relations (car/car alignment). There were also a few orientation relations added, though they were only slightly better than noise (roof/road orientation) and could probably be weeded out by adjusting $\epsilon_{1}$.

Figure 11 shows samples from our final model $p(p g ; \Theta, R)$. The resulting images appear similar to true aerial images, with objects obeying many of the same spatial and appearance constraints that we observe in the real data. We see cars appearing on roads, roofs arranged in blocks, and few or no spurious overlaps. Note that these samples are not representative of a specific aerial image from the
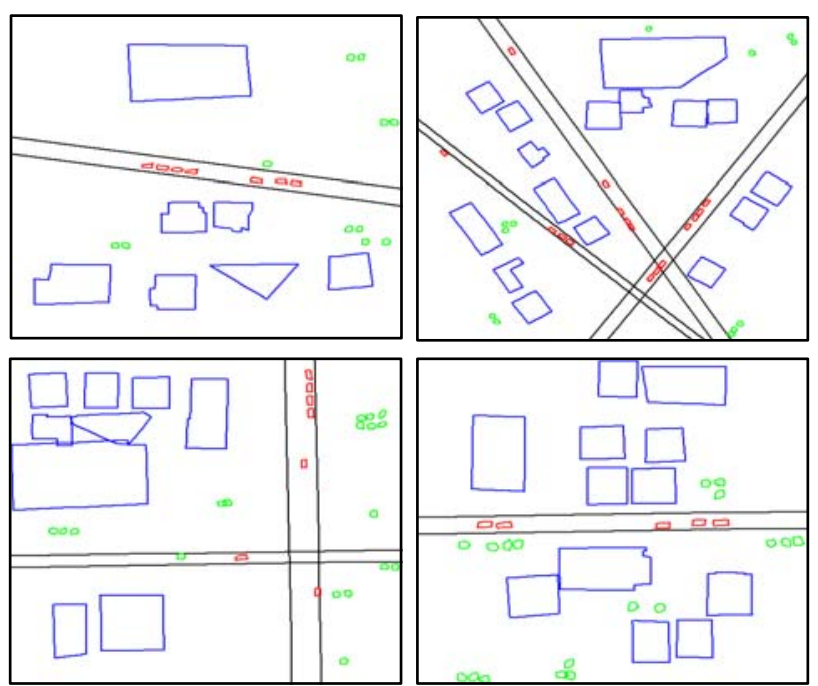

ing the statistics of our learned model. We can create a vast amount of unique object configurations even though we've never observed them directly

training data or elsewhere. These are simply object boundaries that have been scaled, positioned, and oriented such that they minimize the energy in our prior. Nevertheless, we see that the relationship histograms match between the two models and the sampled images are perceptually similar to true aerial images. This shows that our learned model is in fact capturing the relationship statistics present in true aerial images and can thus recreate believable aerial image configurations.

\section{Bottom-Up/Top-Down Bayesian Inference}

In this section we present the Bayesian formulation for finding the highest probability explanation of an aerial image. 
We first run bottom-up detectors for each of the object categories. After this first stage of object detection, we use a cluster sampling algorithm inspired by Swendsen-Wang sampling called $\mathrm{C} 4$ to prune out false positives and inconsistent detections. In the third stage of the algorithm we propose locations of objects that may have been missed by the bottom-up detectors or incorrectly pruned in the first stage.

\subsection{Bottom-Up Detections}

We use different bottom-up detectors for each type of object:

Cars We trained a discriminative AdaBoost classifier (Freund and Schapire 1997) to detect cars. We collected 3000 positive examples of cars, selected by hand as patches containing a single car from aerial images, as well as 3000 negative images for training, comprised of patches of training images in which no car is present. Figure 12(a) shows car detections using the learned classifier. Unfortunately, we do find that this method results in many false positives, which we will address later. AdaBoost is a commonly cited and described algorithm, so we refer the reader to Freund and Schapire (1997) for further details.

Parking lots and Trees Parking lots and trees are characterized by their textures. Color information is highly variable from one parking lot or grove of trees to the next, so color histograms are too simple to capture an appearance model for these classes. We resolve this problem by using
TextonBoost (Shotton et al. 2006), an algorithm for combining texture and shape cues in a boosting framework to create a discriminative classifier. TextonBoost extracts textons (collections of filter responses) for each category and clusters them into a texton dictionary. These textons are then boosted using to arrive at a combined discriminative classifier. We provided TextonBoost with about 100 images in which the images are labeled $(0 / 1)$ according to whether or not a pixel belongs to background or the category we're learning (parking lots and trees are learned separately). The pure bottom-up results are shown in Figs. 12(b) and 12(c).

Roofs and Roads Roofs and roads present quite a different problem from the categories we've represented up until now. They are neither defined by a constant shape nor a constant texture. The most informative cues are the edges that define their boundaries. We use a recently developed algorithm called Compositional Boosting (Wu et al. 2007) that hierarchically combines low-level cues into higher-level structures. A more detailed explanation of Compositional Boosting is given in Wu et al. (2007), but we will describe it at a high level here.

High-level Description of Compositional Boosting Compositional Boosting learns a model by first defining a dictionary of low-level features (such as edges) along with some spatial rules of interest (e.g. parallelism, relative length, collinearity). These low-level features are first labeled in a number of training images and labeled as belonging to the

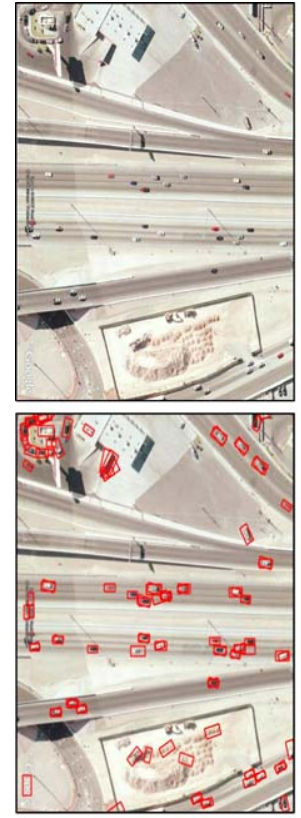

(a) Cars (Adaboost)
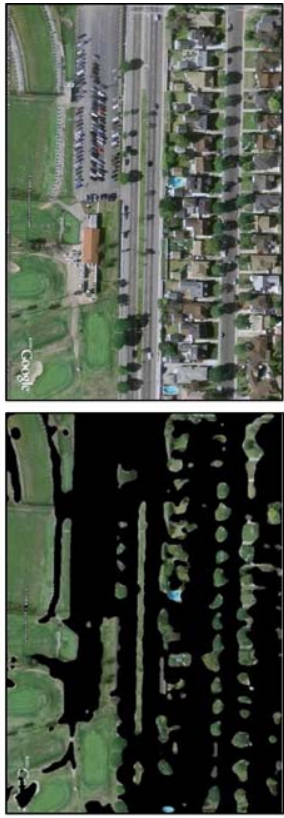

(b) Trees (TextonBoost)

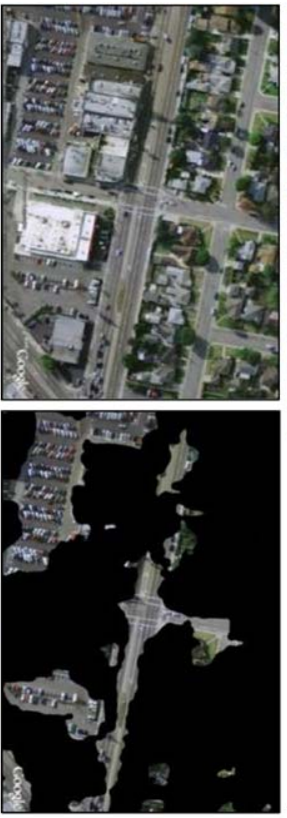

(c) Parking Lots (TextonBoost)
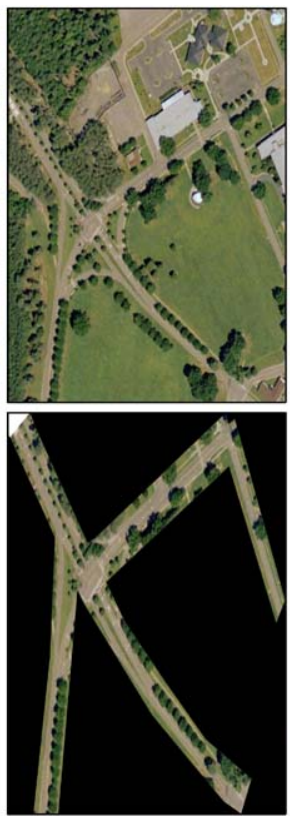

(d) Roads (CompBoost)

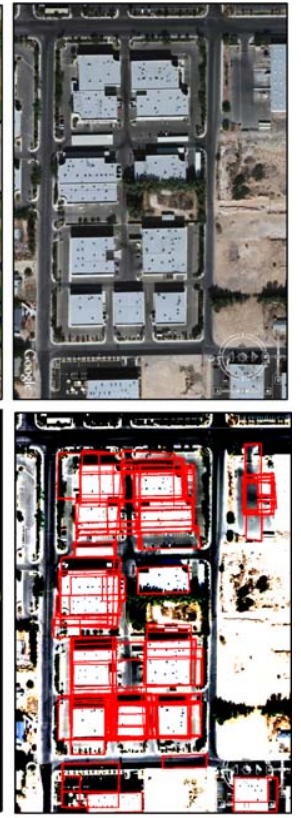

(e) Roofs

(CompBoost)

Fig. 12 Single object detections using our bottom-up detectors 
structure of interest or not. For example, in this experiment, we labeled edges in the training data as belonging to a roof, a road, or neither. Compositional Boosting begins building a hierarchy from these labeled edges by testing the mutual information of edges under certain spatial constraints. For example, in the roof class we will see lines at right angles more frequently than in random noise. Any composition rules with mutual information greater than some threshold are added to the hierarchy (e.g. two lines nearly 90 degrees from one another should form a higher-level component). This process then repeats at the next highest level until some percentage of the labeled lines are modeled by the final composition. Figure 2 shows the Compositional Boosting hierarchies below the roof and road nodes. A roof can decompose into a number of different shapes, each of which is formed from lower-level components.

To detect structures in images, we first define detectors for Compositional Boosting. We begin with an edge detector for edges, since they are the lowest level nodes in our hierarchy. However, we may also define higher level detectors to find higher-level nodes (e.g. corner detectors). Let us define a possible set of detectors $T=\left\{t_{i}: i=1,2, \ldots, k\right\}$ at each node designed to detect that part directly from the image. We also add auxiliary data structures to each node, called "Open" and "Closed" lists. The open lists will store any current potential detections for that node. The closed list will store any accepted detections of the node the list resides at. Each proposal in an open list is weighted by a posterior probability ratio.

Compositional Boosting first creates proposals for the open lists for an image $I$ in one of two ways:

(1) Proposals for $A$ are formed from local detectors $T$. The weight of each detection is the log-ratio of the local marginal posterior probability on an image patch $\Lambda^{i}$ using some features of the image $F()$,

$\hat{\omega}_{A}^{i} \approx \log \frac{p\left(A^{i} \mid F\left(I_{\Lambda^{i}}\right)\right)}{p\left(\bar{A}^{i} \mid F\left(I_{\Lambda^{i}}\right)\right)}$,

where $\bar{A}$ is an alternative hypothesis.

(2) Proposals for $A$ are formed by combining proposals for $A$ 's children from their Open and Closed lists. Proposals from each list are compared based on their compatibility, and highly compatible proposals are combined to propose the higher level node $A$. The weight on these hypotheses is the local conditional posterior probability ratio. Suppose a proposal $A^{i}$ is formed from three of its child proposals $A_{1}^{i}$, $A_{2}^{i}$, and $A_{3}^{i}$, then the weight will be

$\hat{\omega}_{A}^{i} \approx \log \frac{p\left(A_{1}^{i}, A_{2}^{i}, A_{3}^{i} \mid A^{i}\right) p\left(A^{i}\right)}{p\left(A_{1}^{i}, A_{2}^{i}, A_{3}^{i} \mid \bar{A}^{i}\right) p\left(\bar{A}^{i}\right)}$

where $\bar{A}$ represents a competing hypothesis. In other words, we are measuring the probability that these proposals appeared as a result of $A$ existing as opposed to some other node. The top-down process then greedily adds proposals from the Open lists to the Closed lists and updates the Open list weights until no weights are above a certain threshold.

Figure 13 shows a toy example of this process. Here we see that $A$ can be formed from either its detections $T$, or by combining proposals from its children. This is where Compositional Boosting is particularly powerful, because weak detections of compatible children may be enough for us to propose the parent node.

Figure 14 shows an example of roof detection using Compositional Boosting. We begin with a probabilistic edge map formed from our source image. From this map we first extract edge segments using an edge detector. We next combine edges that are compatible according to the rules that

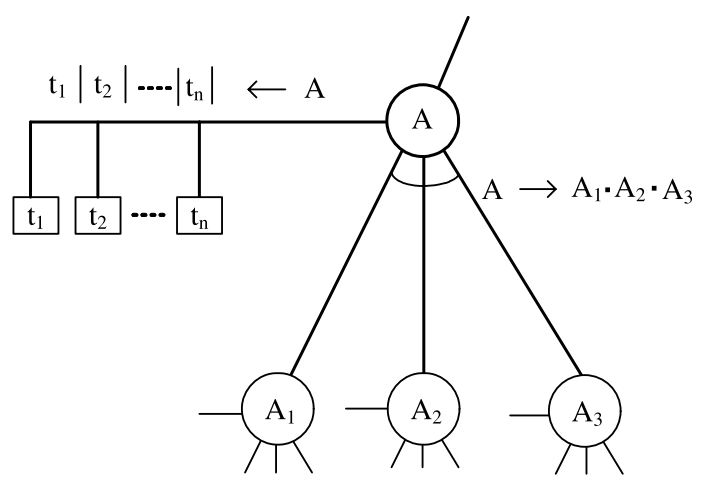

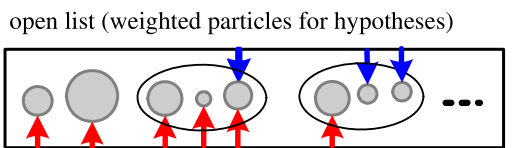

closed list (accepted instances)

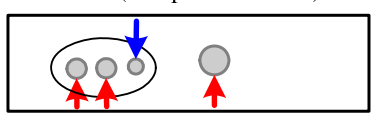

Fig. 13 (Color online) A conceptualization of inference with Compositional Boosting. The left hand side shows an example of a node in a Compositional Boosting tree with parent node $A$ and children nodes $\left(A_{1}, A_{2}, A_{3}\right) .\left(t_{1}, \ldots, t_{n}\right)$ indicate proposals for $A$ detected directly from the image, while $A=A_{1} \cdot A_{2} \cdot A_{3}$ indicates proposals for $A$ detected as a product of child proposals. In the inference process, we store proposals at node $A$ in open and closed lists, where particles in the open list are pending proposals and particles in the closed list have been accepted. The up and down arrows in the figure indicate that there is evidence for each particle coming from both bottom-up and top-down channels 


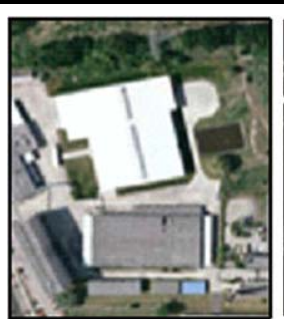

(a)

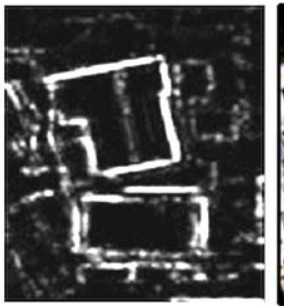

(b)

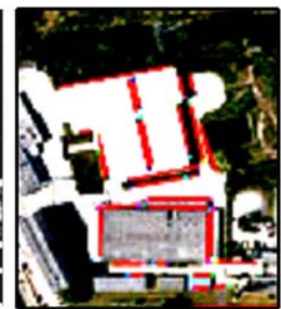

(c)

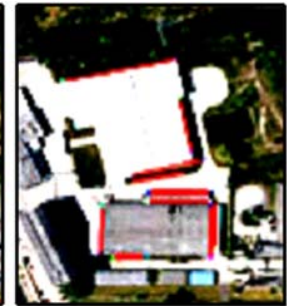

(d)

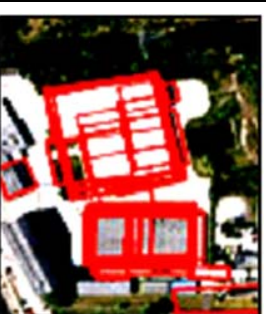

(e)

Fig. 14 An example of detecting roofs with Compositional Boosting. We run a probabilistic edge detector to get image (b), after which the algorithm detects object parts, such as parallel lines and corners in (c) and (d). These act as evidence for the final roof proposals in (e)

we've learned from labeled roofs. Figure 14(c) shows parallel lines detected in the image, while Fig. 14(d) shows corners detected in the image. Figure 14(e) shows the final roof detections inferred from the composition of these low-level features. We can see that the rectangular structures of roofs are detected, but there are also many false positives present.

\subsection{Top-Down Bayesian Formulation}

Using the approach above, we arrive at a set of $N$ candidate proposals, $C_{0}=\left\{c_{i}: i=1,2, \ldots, N\right\}$. Each candidate proposal consists of a boundary of an object that was detected using bottom-up detectors. We also use each detector's output as a measure of the object likelihood, $L_{i}=p\left(I \mid b_{i}, l_{i}\right)$. The computation of this likelihood varies from detector to detector, but in our experiments, for example, we computed color histograms for classes like trees, parking lots, and roads and used those to compute the likelihood of a bounded region based on its color distribution. Other methods like Compositional Boosting return a probability for the detected object, which we used as its likelihood score. For our AdaBoost candidates we used the number of overlapping proposals for each object as a measure of the likelihood of the object. Note that each likelihood is independent of the other classes, i.e. $L_{i}$ measures the probability that a patch belongs to class $A$ versus that it doesn't, not the probability that it belongs to class $A$ versus class $B$. Ideally we would find a multiclass model that accounted for the probability that a patch belonged to class $A$ over class $B$ or class $C$, but for now we use a simple two-class approximation to measure the strength of each proposal. Each proposal is then $c_{i}=\left\{b_{i}, l_{i}, L_{i}\right\}$.

Our goal is to find the parse graph $p g$ that best describes the image. $p g$ will consist of a set of candidates $C \subseteq C_{0}$ and grouping decisions such that the likelihood of the candidates and probability of their configuration is maximized. The Bayesian formulation is

$$
p g^{*}=\underset{p g}{\operatorname{argmax}} p(p g \mid I)=\underset{p g}{\operatorname{argmax}} p(p g ; \Theta, R) p(I \mid p g)
$$

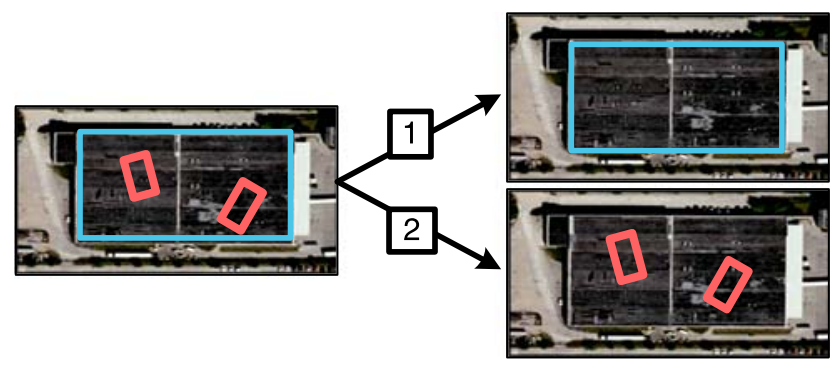

Fig. 15 An example of two choices a greedy inference algorithm could make for interpretations of the scene. Because cars cannot appear on top of roofs (let us assume the data supports this) selecting the roof in decision 1 eliminates the car nodes while selecting the cars in decision 2 does the opposite. The algorithm is stuck with this decision no matter what later evidence it may find

\subsubsection{Previous Approach and Motivation}

The conference version of this work (Porway et al. 2008) used a greedy algorithm to maximize this posterior. In that work, the authors used an iterative approach that first assigned a weight $\omega\left(c_{i}\right)$ to each of the currently unselected proposals based on how well it maximized the posterior. The object with the highest weight was selected to be added to the running parse of the scene, $p g$, thus forming $p g_{+}$. The objects were then reweighted according to how much the remaining objects would improve the overall explanation of the scene and this process iterated until no objects above a certain weight remained.

The problem with this approach is shown in Fig. 15. Because the algorithm described in Porway et al. (2008) is greedy, it cannot backtrack from a poor decision. By selecting the car node to be in the final parse of the scene in the second case, the algorithm will now give an exceedingly low weight to the enclosing roof, as we virtually never see cars on top of roofs. However, had the roof been selected first, as in scenario 1 , the car would have been given a very low weight and we would have arrived at the correct interpretation. We would like our new algorithm to be able to backtrack from these mistakes. 


\subsubsection{Motivation: Swendsen-Wang Clustering}

One solution to this problem is to use Swendsen-Wang clustering (SWC). SWC was designed to sample the Ising model by updating the labels of many sites at once instead of using Gibbs sampling to update one at a time (Barbu and Zhu 2005; Swendsen and Wang 1987). This is similar to our task of switching many objects in or out of the explanation at once. We'll look at SWC at a high level to motivate our solution to the problems in our specific task.

In classic Swendsen-Wang we have a number of sites with class labels,

$X=\left\{x_{1}, x_{2}, \ldots, x_{n}\right\}, \quad l\left(X_{i}\right)=\left\{l_{1}, l_{2}, \ldots, l_{k}\right\}$,

that have a neighborhood structure of edges $E$ connecting them. The goal of the algorithm is to assign the labels such that some energy term $\xi(X)$ is minimized. In the Ising model, this is simply a constraint that neighboring sites have the same label

$$
\begin{aligned}
& p(X)=\frac{1}{Z} \exp ^{-\xi(X)} ; \\
& \xi(X)=\beta \sum_{\langle s, t\rangle \in E} \delta\left(l\left(x_{s}\right)=l\left(x_{t}\right)\right), \quad \beta>0 .
\end{aligned}
$$

In our model, $p(X)$ would be our learned prior $p(p g ; \Theta, R)$. SWC updates portions of $X$ quickly by clustering neighboring nodes that have the same color and updating their labels simultaneously. It does this by first introducing an auxiliary variable into the Ising model, $U$, indicating whether an edge is "on" or "off" between two nodes,

$U=\left\{u_{s t}:\langle s, t\rangle \in E\right\}, \quad u_{s t} \in\{0,1\}$.

Edges are turned on with probability $\rho$ if the nodes they connect have the same value, and are turned off otherwise.
These edges will be used to join nodes with the same labels into connected components.

The benefit of adding $U$ to the formulation to get $\pi(X, U)$ was that Swendsen and Wang could now take large steps in the solution space. It was shown in Swendsen and Wang (1987) that sampling from $\pi(X \mid U)$ and $\pi(U \mid X)$ iteratively produced samples from $\pi(X, U)$. If this distribution was defined such that we can marginalize over $U$ to get $p(X)$ back, then we can generate samples from $p(X)$, which is difficult and slow, by instead sampling from $\pi(X, U)$, which is easy and fast.

Without delving too deeply into the technical details, Swendsen and Wang's algorithm did just that in the following algorithm:

1. Sample from $\pi(U \mid X)$ to turn edges on and off. Edges between nodes with different labels are turned off w.p. 1 . Edges between nodes with the same label are turned off w.p. $1-\rho$, where $\rho=1-e^{-\beta}$ for the Ising model.

2. Form connected components $C C P$ based on the edges that are left on at this iteration. All nodes within each $C C P$ have the same label by the definition of $\pi(X \mid U)$.

3. Select a connected component $C C P_{i}$ at random.

4. Sample from $\pi(X \mid U)$ to relabel the nodes in $C C P_{i}$ according to the state of neighboring nodes. This quantity is often computed by Gibbs sampling the probabilities of every possible labeling.

It can be shown that, because $p(X)$ can be derived from $\pi(X, U)$ by summing over $U$, we can sample from $p(X)$.

Figure 16 shows an example of running SwendsenWang clustering on the Ising model. In Fig. 16(a) edges are probabilistically turned on or off based on the labels of the nodes they connect to. This is equivalent to sampling from $\pi(U \mid X)$. A connected component is then formed in Fig. 16(b) based on the edges currently on and its labels are

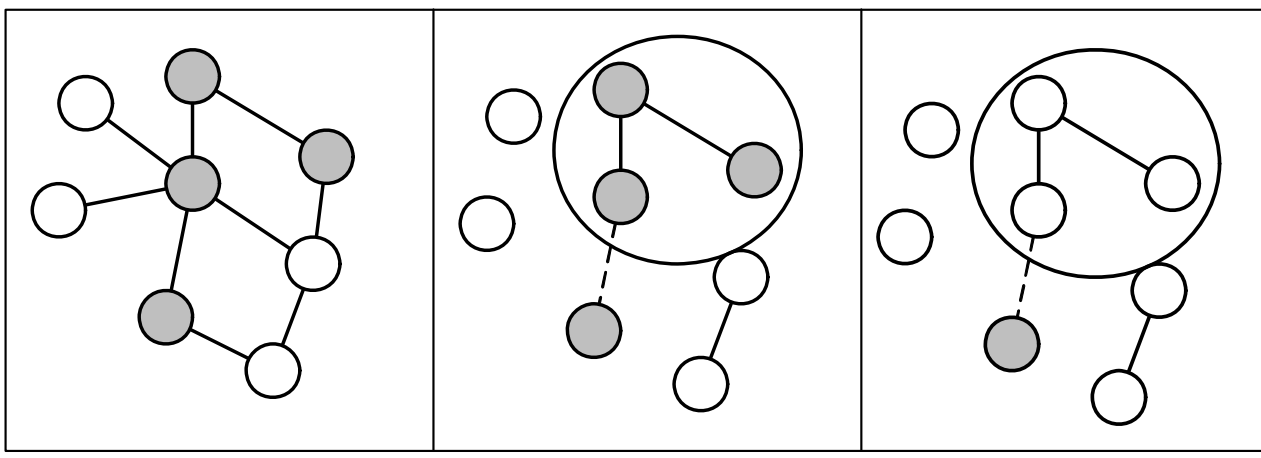

(a) Initial state

(b) Edges cut probabilistically

(c) Component recolored

Fig. 16 An example of Swendsen-Wang clustering on the Ising model. (a) The current state. (b) Nodes with different labels have their edges turned off, while nodes with the same label are connected w.p. $e^{-\beta}$. The dashed lines show edges that were probabilistically cut between nodes with the same label. The remaining edges form connected components. (c) Simultaneously relabel all nodes in a randomly selected connected component. This process updates large portions of the solution space quickly 
reassigned in Fig. 16(c). This last step is equivalent to sampling from $\pi(X \mid U)$. The end result from this process are samples from $\pi(X, U)$, and thus $p(X)$.

This approach will not work for our task, however, for two main reasons:

1. Alternative explanations: In our task, each object proposal $c_{i}$ can take a label $l_{i} \in\{0,1\}$, indicating if it is in the current explanation or not. If we label a cluster using traditional SWC, we can remove a cluster of proposals from our explanation without adding any nodes back in. This may cause our posterior probability to decrease, as we have now explained less of the image. With no alternative explanation to replace these removed proposals we simply decrease the probability of our current system, which means this move is accepted with a very low probability.

2. Conflicting nodes: If we add an object to the solution set that conflicts with other objects, say a car on top of a tree, this will give the explanation such high energy that this move will never be accepted. This is the opposite problem from problem 1, in that we now need to remove parts of the current explanation simultaneously with adding the new explanation, otherwise the combined explanation might have a very low probability.

An example of this problem is shown in Fig. 4. We need an algorithm that can exchange alternative explanations simultaneously without introducing conflicting objects to the explanation.

\subsection{C4: Clustering via Cooperative and Competitive Constraints}

We use an algorithm called Clustering via Cooperative and Competitive Constraints (C4) (Porway and Zhu 2009) to deal with these problems. It differs from Swendsen-Wang clustering in two major ways:
1. Negative edges: In addition to the "positive" edges in Swendsen-Wang clustering, in which nodes were encouraged to have the same label, $\mathrm{C} 4$ incorporates negative edges, dictating that neighboring sites should not be labeled similarly. We use them here to indicate that two explanations of the scene can't both exist at once. For example, we could have negative edges between two overlapping cars to indicate that they cannot both be in the same explanation at the same time.

2. Composite Flips: Traditional Swendsen-Wang updates the label of a single cluster in one step. In our model the new labels for one cluster may cause it to violate constraints with neighboring clusters, so we may need to update the labels of many clusters simultaneously. We thus form composite components consisting of conflicting clusters that all need their labels reassigned at once to remain consistent. This is like the switch shown in Fig. 4(c), where an entire parking lot and cars are not only taken out of the candidate set, but are replaced with a roof simultaneously.

Figure 17 shows C4's results on a toy model. In this example, we have introduced a backbone of negative edges down the middle of the lattice, requiring that nodes on one side have the same color, but each side has a different color. Traditional Gibbs sampling attempts to update one site at a time, which creates a low probability state. SWC updates an entire side at one time, but only updates one cluster and ignores negative edges, thus creating another low probability state. C4 clusters the entire system and relabels the individual clusters subject to both positive and negative constraints, creating a high probability state.

We can extend the example in Fig. 17 to our actual problem. Figure 18 shows a number of object proposals, which are like the nodes in Fig. 17. Objects that have a high prior probability of being on together are grouped together with

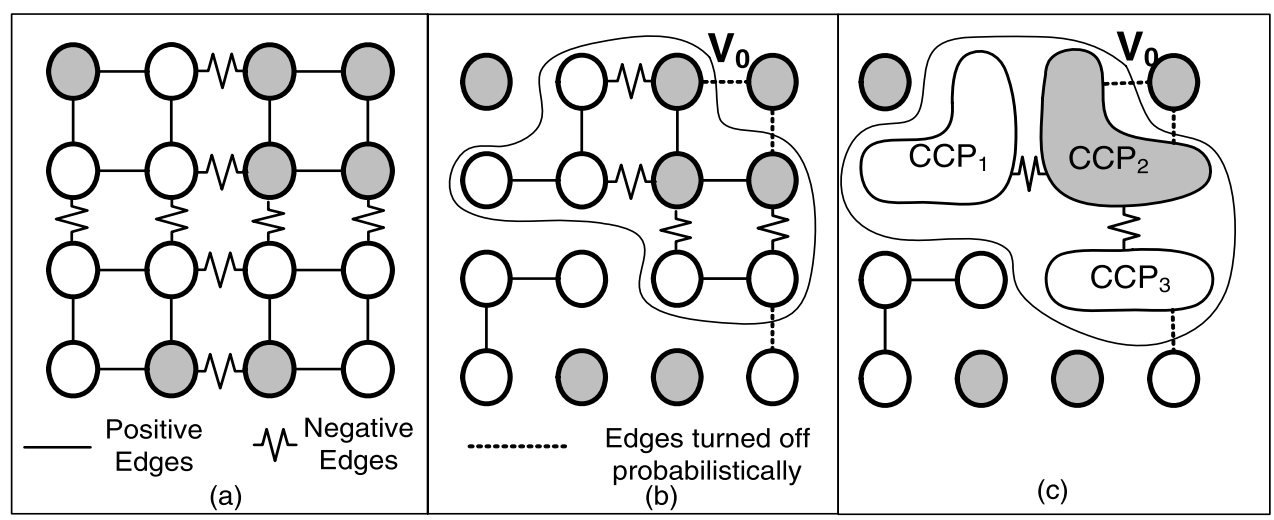

Fig. 17 A toy example of $\mathrm{C} 4$ on the Ising model. Here we've added negative edges, indicated by jagged lines, that encourage nodes to have opposite labels. In (b), we probabilistically turn edges on and off based on whether they satisfy their current constraints (node labels same or different). The remaining edges form connected components (CCPs). (c) A connected component $V_{0}$, which consists of sub-components $C C P_{i}$ of the same label connected by negative edges, is relabeled according to its constraints. Further details are provided in Porway and Zhu (2009) 


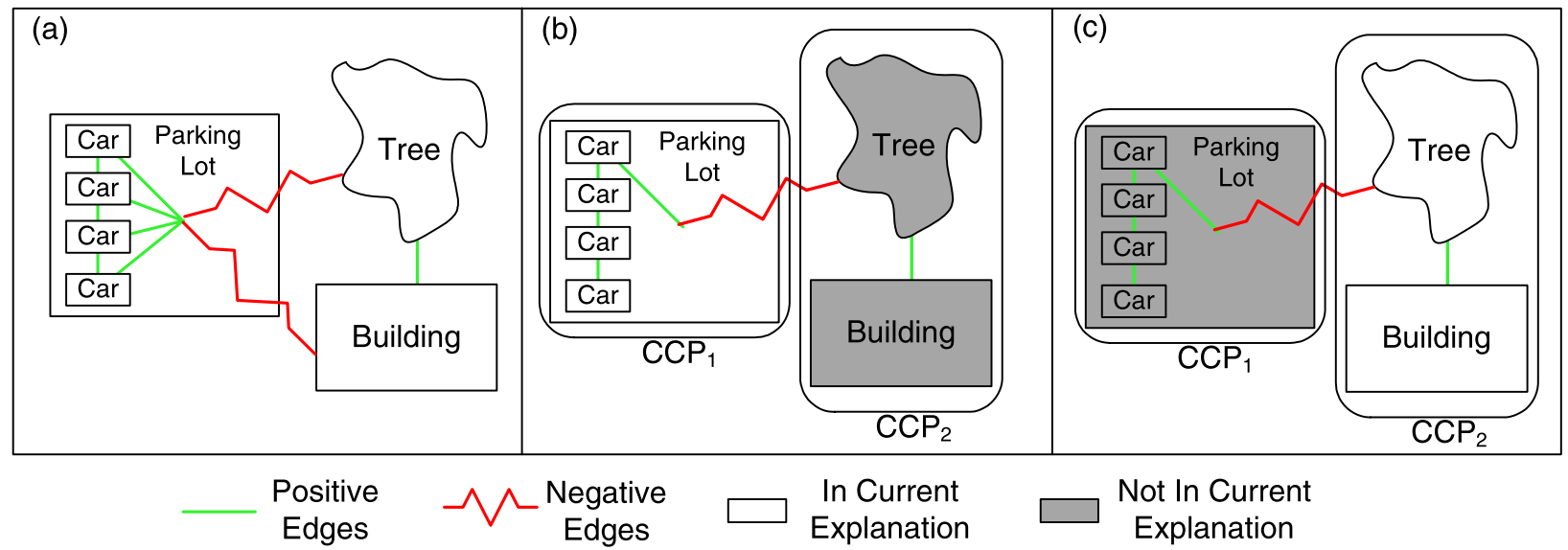

Fig. 18 An application of $\mathrm{C} 4$ to a toy aerial image. (a) Compatible object proposals (cars in the parking lot, the tree next to the building) are connected by positive edges while non-compatible proposals (tree with parking lot, building with parking lot) are connected by negative edges. (b, c) $\mathrm{C} 4$ groups objects based on these connections and updates the state of the system to swap between alternate explanations "positive" edges (e.g. the neighboring cars and the parking lot, the tree with the building), while objects that have low prior probability of being on together are grouped by "negative" edges (e.g. the building with the parking lot or tree with parking lot). Here we've added positive and negative edges based on pairwise energies from the exponent in $p(p g ; \Theta, R)$. If the energy between two nodes is above a certain threshold, a negative edge is added between them. Otherwise, a positive edge is added.

The probability assigned to each edge is determined by using a squashing function of the energy between the two nodes. This is used to map the pairwise energy to the range $[0,1]$. In our experiments we used an inverted logistic function $F(x)=\frac{1}{1+e^{-(x-u) / s}}$ where first $F(x)=1-F(x), x<0$ and then we scale to $[0,1]$ via $F(x)^{\prime}=2 * F(x)-1$. This creates a symmetric function where energies that are much greater or much less than the center of the distribution $u$ approach probability 1 , while values near $u$ approach 0 , as they are just barely positive or negative. The parameters $u$ and $s$ can be adjusted to translate and scale the function, respectively. In this way we create data-driven probabilistic edges between proposals based on their pairwise energies.

In the same way that it does in Fig. 17, C4 probabilistically turns positive and negative edges on and off, then proposes to relabel the selected cluster based on its constraints, giving a label of 0 to objects that shouldn't be in the current interpretation and a label of 1 to objects that should be in the current interpretation. Here $\mathrm{C} 4$ flips between the two possible explanations of the scene in Figs. 18(b) and (c). The decision to flip the interpretation or not is reached by Gibbs sampling the possible component values (here 0 or 1 ), meaning that each interpretation is selected to be turned on proportionally to the value of $p(X)$ it results in. In the case of two equally likely interpretations, there is a $50 \%$ chance that the interpretation will swap at each step. At the conclusion of this process (which is sampling from $\pi(U \mid X)$ and $\pi(X \mid U)$ as in SWC) our samples from $p(p g \mid I)$ are sets of proposals for interpreting the scene. Further derivations and results can be found in Porway and Zhu (2009).

\subsection{Top-Down Prediction}

In addition to reducing false positives using $\mathrm{C} 4$, we can also predict new instances of objects that may have gone undetected using the hierarchical aspect of our model.

Given our final parse $p g^{*}$ from C4, we can Gibbs sample the group nodes to create new objects per group node or new group nodes in the scene. We can then Gibbs sample the appearance relationships for newly added nodes to form new proposals. As many objects are loosely constrained in the scene it is difficult to predict where objects should be exactly. We remedy this by just predicting additional aligned objects wherever we already have some aligned objects. This allows us to find new true positives in addition to pruning false positives. Figure 5 shows examples of hallucinated objects using the results from the $\mathrm{C} 4$ pruning. These final proposals will be added to a final round of $\mathrm{C} 4$ clustering to arrive at the final parse.

\section{Experiments}

We ran our algorithm on 5 large $(4000 \times 4000)$ images collected from Google Earth. We learned a top-down model as in Sect. 4 and implemented detectors for each of the objects as described in Sect. 7.1. Figure 19 shows the process of our algorithm on one of these images. The first panel shows the original image, while the second panel shows an overlay of the initial bottom-up detections, which contains a huge 


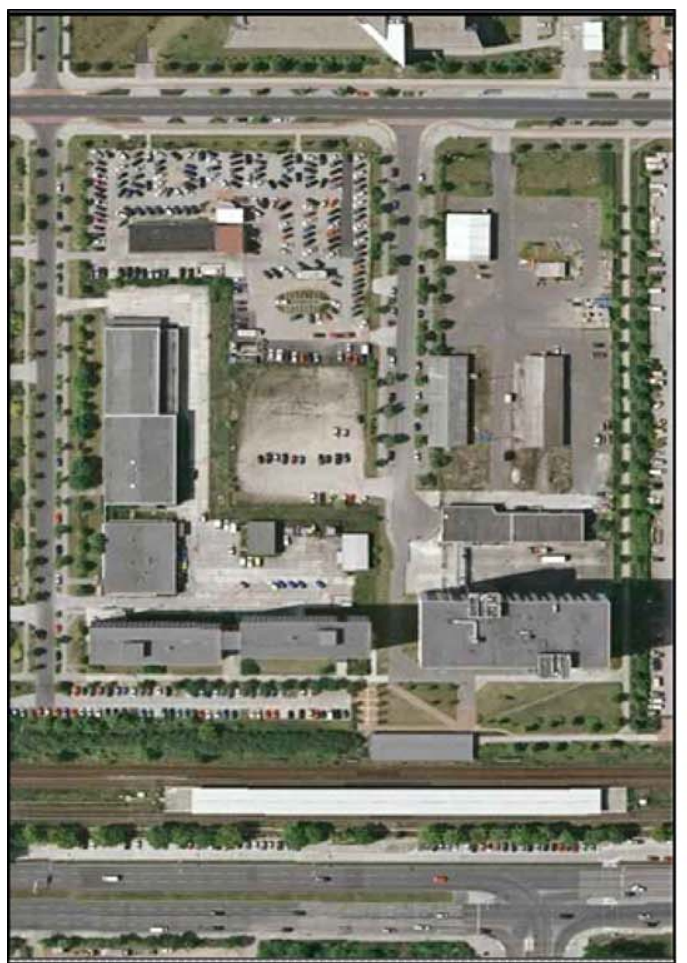

(a)

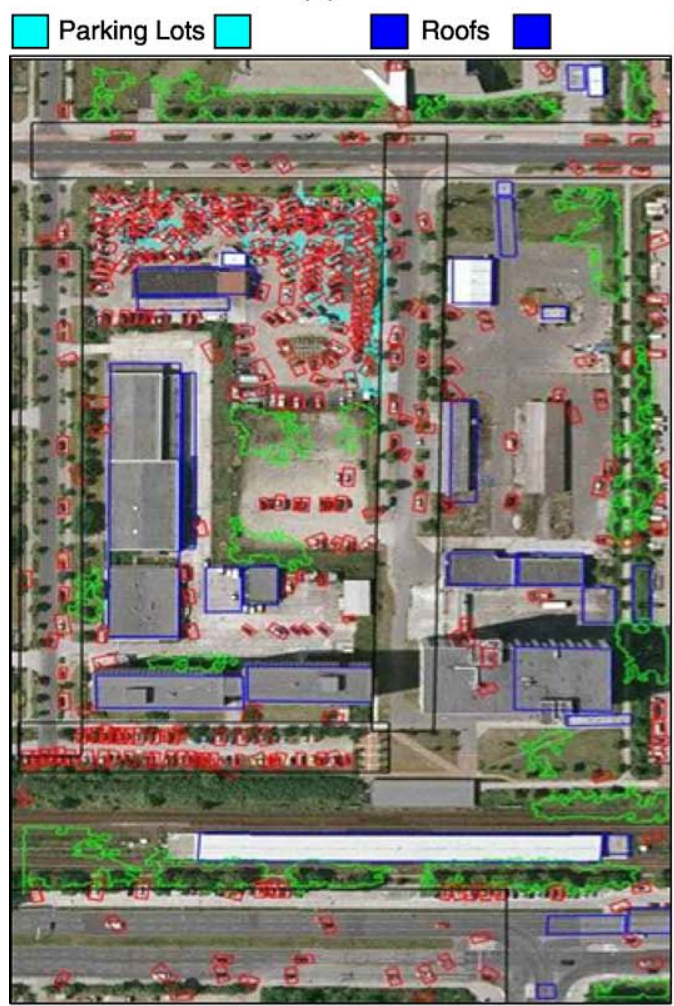

(c)

Fig. 19 The bottom-up to top-down pipeline. (a) The original image. (b) The bottom-up detections. There are a huge number of overlapping and inconsistent detections. (c) The top-down pruning results us-

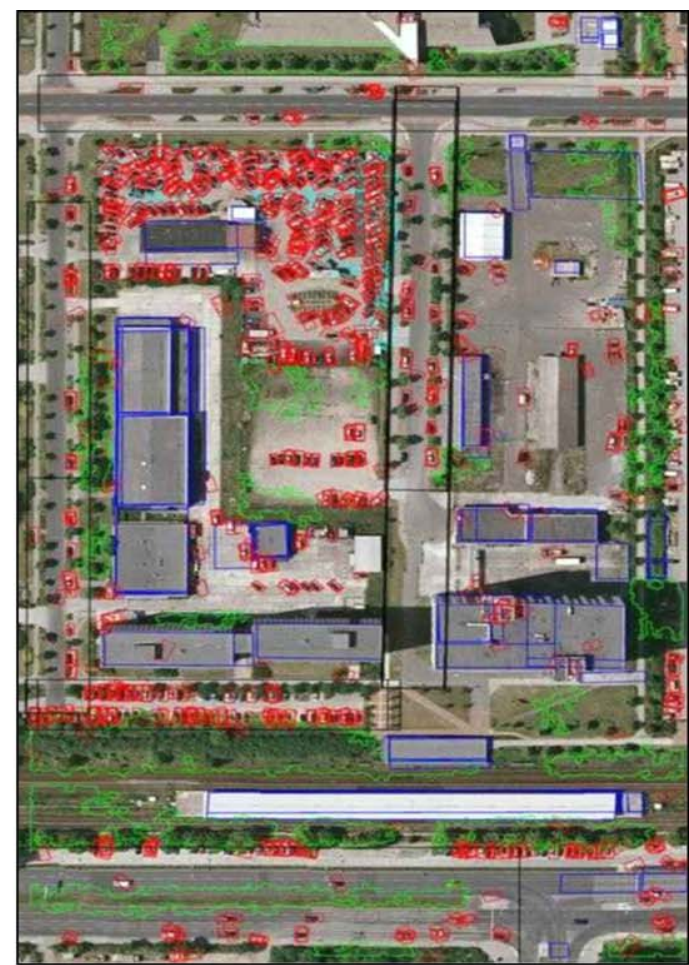

(b)

Cars

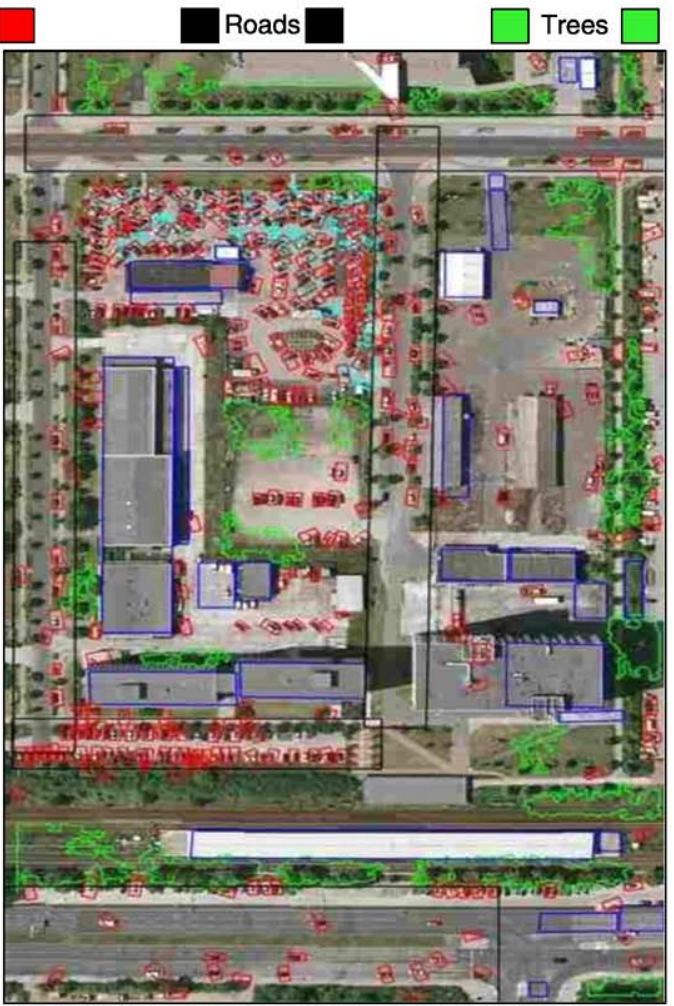

(d)

ing C4. Many false positives are removed. (d) The results given newly proposed nodes from the hierarchical prior 


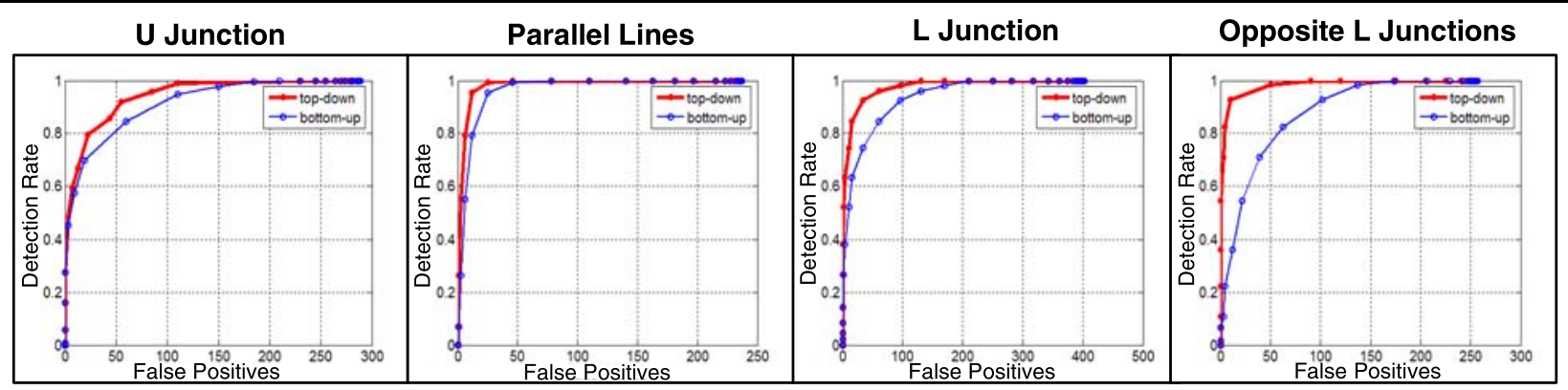

Fig. 20 ROC curves for detecting U junctions, L junctions, parallel lines, and opposing L junctions using Compositional Boosting. We see that CompBoost helps us identify weakly detected junctions, which helps us propose better high-level detections

Fig. 21 Close up views of our improvement during pruning. Notice that overlapping proposals and inconsistent explanations (cars in trees) have been removed

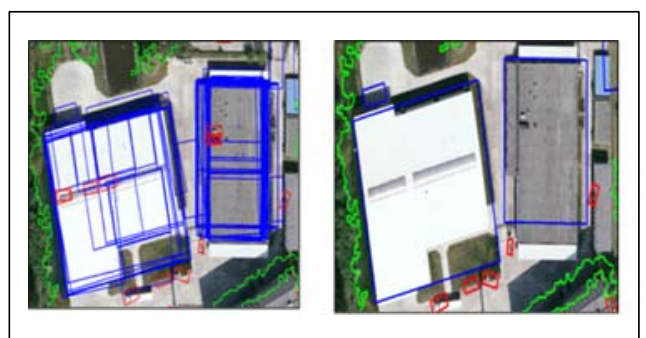

(a)

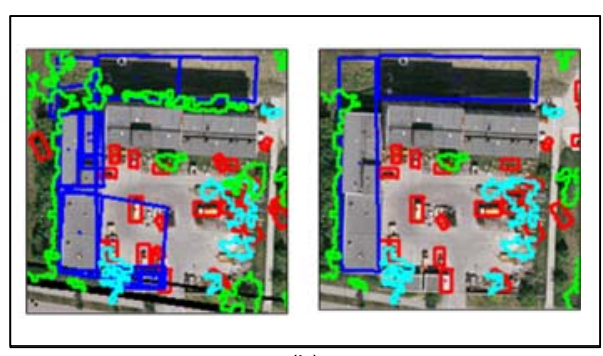

(b) number of false positives ( 3 false positive roads, 71 false positive buildings, 623 false positive cars, 10 false positive trees). The third panel shows the results of using $\mathrm{C} 4$ clustering to find a high probability set of bottom-up detections to explain the scene. The fourth panel shows the final explanation of the image after some new proposals have been suggested and verified. The first step of the $\mathrm{C} 4$ algorithm shows the most dramatic improvement, with vast numbers of inconsistent detections (cars on roofs, trees on roads, overlapping roofs) being removed, leaving just single object boundaries for the important objects (we now have 0 false positive roads, 5 false positive buildings, 57 false positive cars and 0 false positive trees). The second step gives a slight improvement, though primarily just in finding missing cars, which are difficult to see at this resolution. Note that there are still some missed detections, either because our initial detectors did not detect the object or because the context may have inadvertently ruled out a valid explanation (e.g. accidentally favoring the shadow of a roof instead of the roof itself, thus suppressing the true roof).

The inference stage, given bottom-up proposals, takes about 10 seconds to run on a dual core $1.6 \mathrm{GHz}$ machine. The bottleneck in our pipeline is the detection phase, however. For example, our AdaBoost results take a mere couple of seconds to compute. The edge detector we used, on the other hand, can take upwards of a minute to process each image. Therefore, the speed of our approach is highly dependent upon the speed required to compute the initial bottomup detections.
Because of the newness of Compositional Boosting, we first examined how much improvement we achieved in detecting low-level roof parts using Compositional Boosting. Figure 20 shows ROC curves for detecting $\mathrm{U}$ junctions, L junctions, parallel lines, and opposing $\mathrm{L}$ junctions. Using specific bottom-up detectors alone (the blue curves) causes us to miss a lot of the junctions present. By using Compositional Boosting, we are again able to leverage context and hierarchy to identify missing junctions to help us propose more roofs, as shown by the red curves.

Figure 21 shows a zoomed in view of our test images before and after pruning. Figure 21 shows that, at first, we have many conflicting proposals for the object boundaries, notably that a parking lot could be on top of the roof. After we enforce the contextual constraints we learned, however, we return to a sensible explanation of the scene, one in which there are no longer cars on top of roofs or overlapping proposals. Figure 22 shows a zoomed in view after we propose new cars. Initially we missed some cars in the rows of the parking lots. Because our model recognizes that cars appear in rows, however, it proposes cars of roughly the same shape and sizes of the neighboring cars around them, using a line grammar. Cars matching above a certain likelihood are accepted and the conflicting nodes are removed.

Table 3 shows the detection rate and false positives per image of each category using just that category's detector (shown in parentheses) vs. using the full hierarchical contextual model. We can see that the hierarchical contextual model greatly reduces the false positive rate from singleobject bottom-up detectors because it can leverage context 
to remove false positives. Our detection rate is about the same, however, as the pruning phase in the second stage serves mostly to rule out inconsistent detections. In the third stage we were able to identify a few extra cars (as shown in

Table 3 False positives per image and detection rates for bottom-up detectors versus our method

\begin{tabular}{lcc}
\hline Detection method & $\begin{array}{c}\text { False positives } \\
\text { per image }\end{array}$ & Detection rate \\
\hline Cars (AdaBoost) & 242.33 & $88.1 \%$ \\
Cars (Ours) & $\mathbf{7 1 . 8 3}$ & $\mathbf{8 4 . 2} \%$ \\
Parking Lots (TextonBoost) & 1.17 & $84.3 \%$ \\
Parking Lots (Ours) & $\mathbf{0 . 1 6}$ & $\mathbf{8 4 . 3} \%$ \\
Trees (TextonBoost) & 14.5 & $88.8 \%$ \\
Trees (Ours) & $\mathbf{9 . 3 3}$ & $\mathbf{8 8 . 8} \%$ \\
Roofs (CompBoost) & 73.5 & $70.3 \%$ \\
Roofs (Ours) & $\mathbf{1 . 6 7}$ & $\mathbf{7 0 . 3} \%$ \\
Roads (CompBoost) & 5.67 & $95 \%$ \\
Roads (Ours) & $\mathbf{0 . 0 5}$ & $\mathbf{8 8 . 3} \%$ \\
Combined (All Detectors) & 337.17 & $93.1 \%$ \\
Combined (Ours) & $\mathbf{8 3 . 0 4}$ & $\mathbf{8 7 . 5} \%$ \\
\hline
\end{tabular}

Fig. 22), but the amount of extra detections was not enough to account for the inadvertent pruning of true positives from stage 2. Overall, our context allows us to achieve comparable detection rates to single-object detectors, but with far fewer false positives.

Figure 23 shows two different precision-recall curves for the bottom-up and top-down stages of our process. We show precision-recall as opposed to ROC curves because it is difficult to decide how to compute the number of true negatives for multi-category classification tasks, a decision that can drastically alter the appearance of the algorithm's performance. In Fig. 23(a), we measure our accuracy at the pixel level. In this experiment, we labeled each pixel in the image as belonging to an object category or not and then converted our inferred boundaries to a similar labeling. In Fig. 23(b) we measure our performance using object-level accuracy. In this case we considered an object to be detected if it had a boundary around it within some threshold of its true scale and position. In both cases we can see that the topdown improvements over the initial detections are substantial. While the initial detections give average performance, it is the introduction of the top-down pruning and prediction that flattens our curve, enabling us to keep a very high level

Fig. 22 Close up views of our improvement during top-down prediction. Additional cars are added to the rows due to the presence of other collinear cars

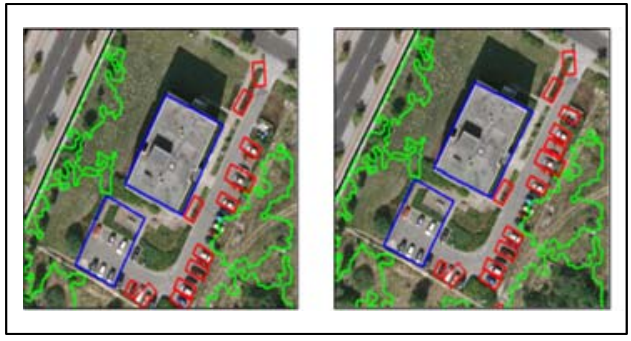

(a)

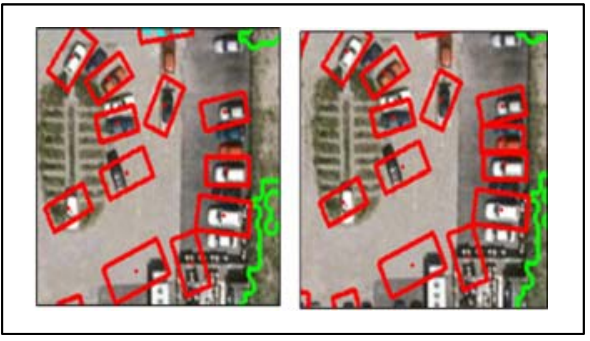

(b)

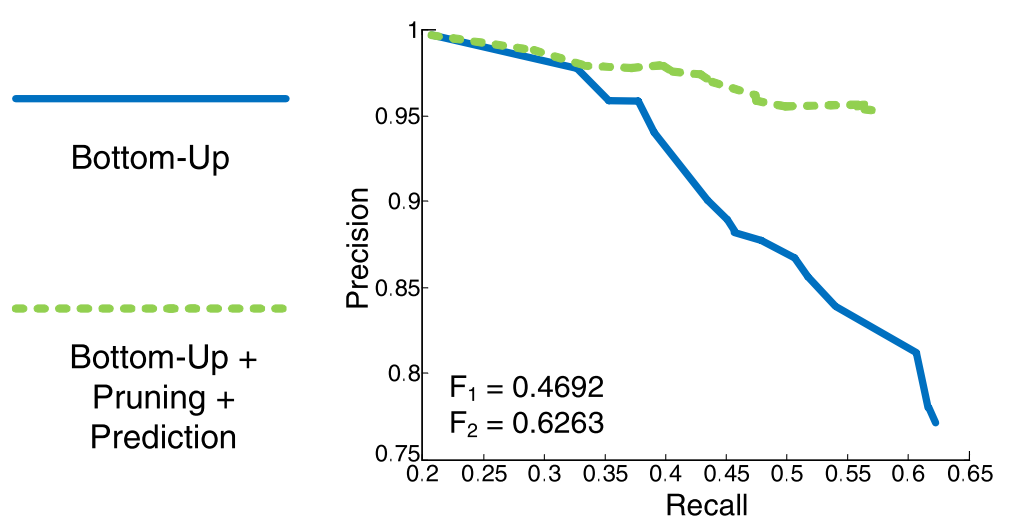

(a)

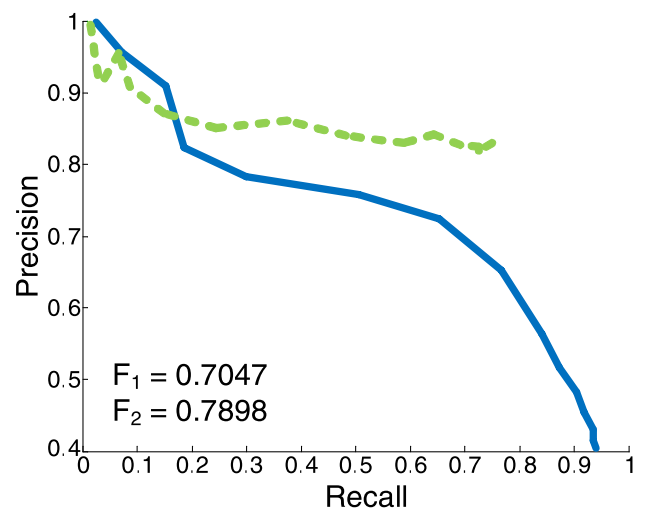

(b)
Fig. 23 Precision-Recall curves for the bottom-up and top-down inference algorithm. In both cases we see a huge improvement by using CSW to prune out false positives and using our model to predict missing objects. $F_{1}$ and $F_{2}$ are the best F-measures for the bottom-up and for the full algorithm, respectively. (a) Precision-Recall curve using pixel-level accuracy, i.e. each pixel in the image is assigned a category label. (b) Precision-Recall curve using object-level accuracy, i.e. each object is considered detected if we infer an object of appropriate dimensions over it 
Fig. 24 An example of swapping alternative solutions using C4. (a) Vents are incorrectly labeled as cars on top of the roof. (b) The cars are correctly swapped out for the roof simultaneously to arrive at the correct solution

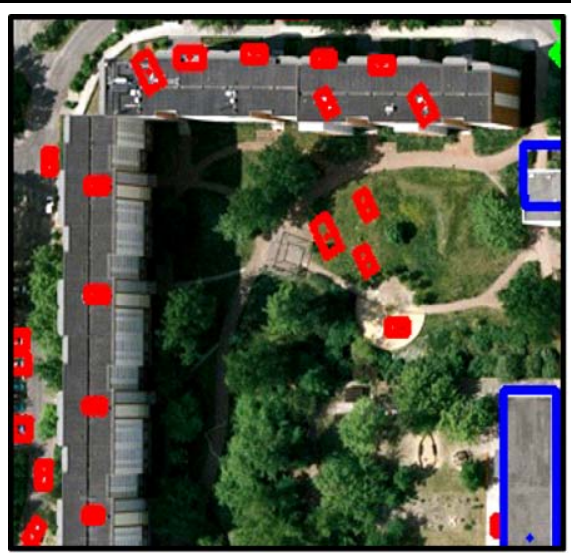

(a)

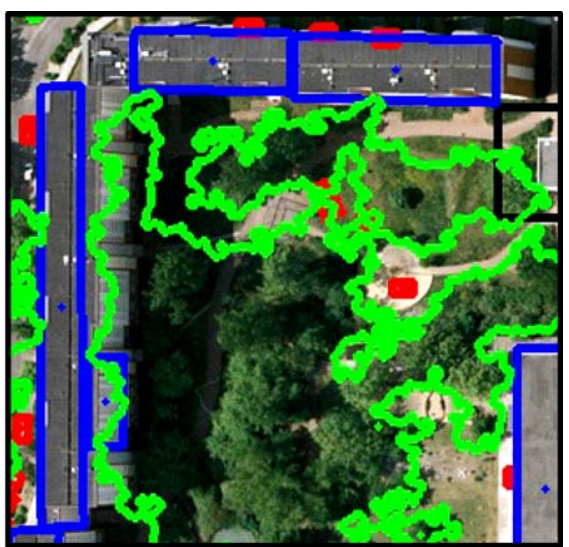

(b)

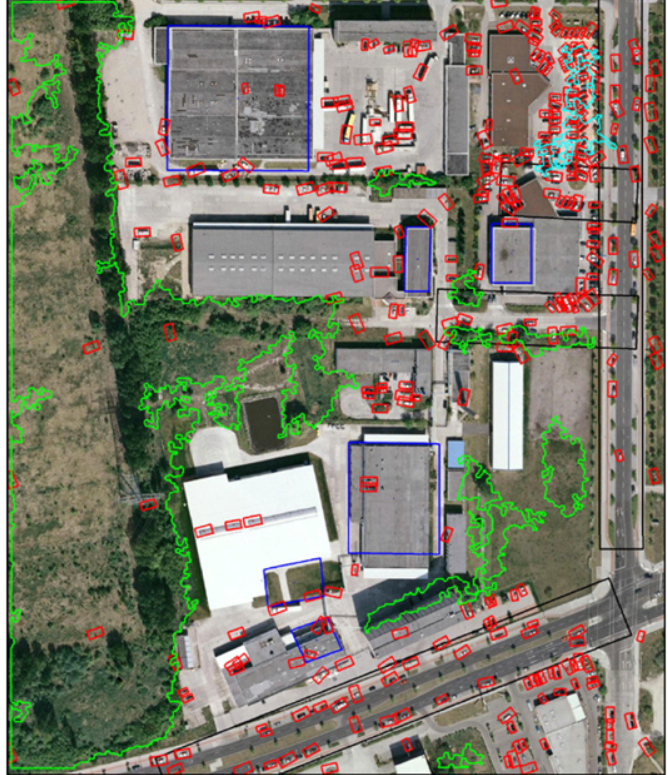

Partially Learned Model
Fig. 25 A comparison of inference results for the model learned with a very high $\varepsilon_{1}$ versus a rather low $\varepsilon_{1}$ (i.e. fewer relationships are added to the first model). The partially learned model is missing a lot of over-

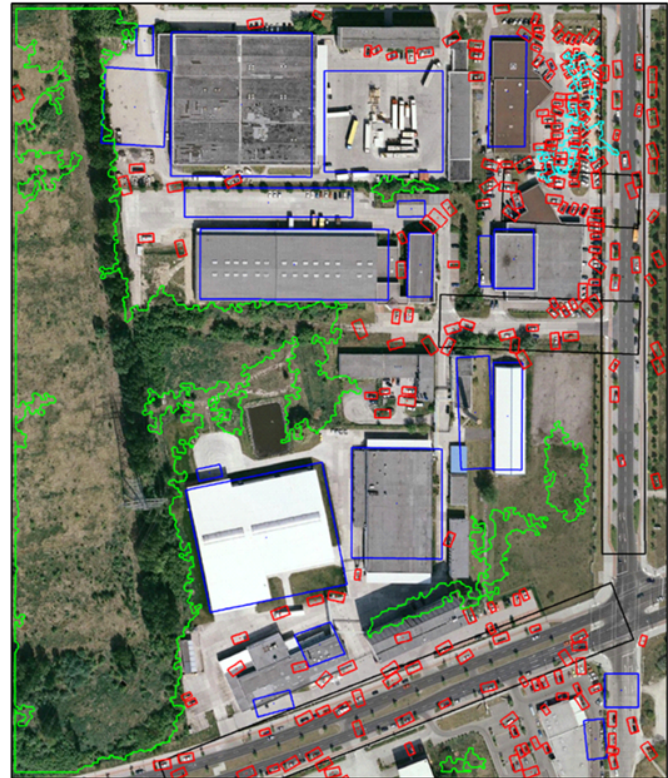

Fully Learned Model

lap constraints (e.g. cars on trees, cars on buildings), and so makes very poor decisions when parsing the scene. Many of the buildings have cars on top of them and cars readily overlap each other

out all of the related cars while simultaneously adding the roof, we are able to arrive at the correct solution in panel (b). This solution is maintained because it has a higher probability than the previous explanation.

In Sect. 4.2 we mentioned that we choose to add relationships iteratively instead of fitting the full model, as this allows us to keep our model simple for sampling and performing inference. The question remains, however, about whether we need to learn as many relationships as we do. Figure 25 shows the inference results for an aerial image using a model with a high $\varepsilon_{1}(10)$ and a model with a standard $\varepsilon_{1}(4)$. We can see that the partially learned model is lacking contextual relationships for cars and buildings, as many cars appear on roofs, and cars appear on top of one that there is a building there. Thanks to C4's ability to swap 

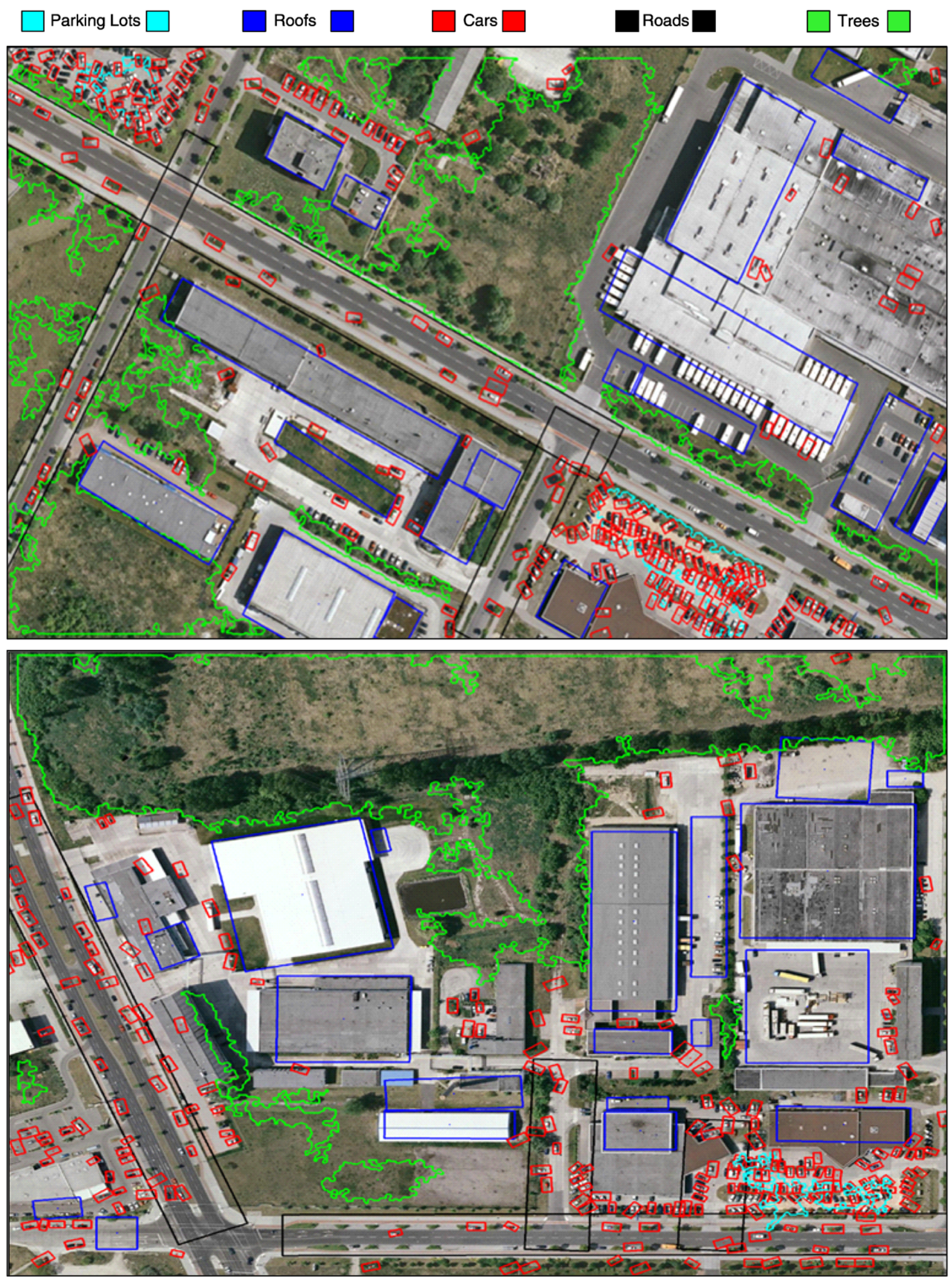

Fig. 26 Flat configurations of parsed images 

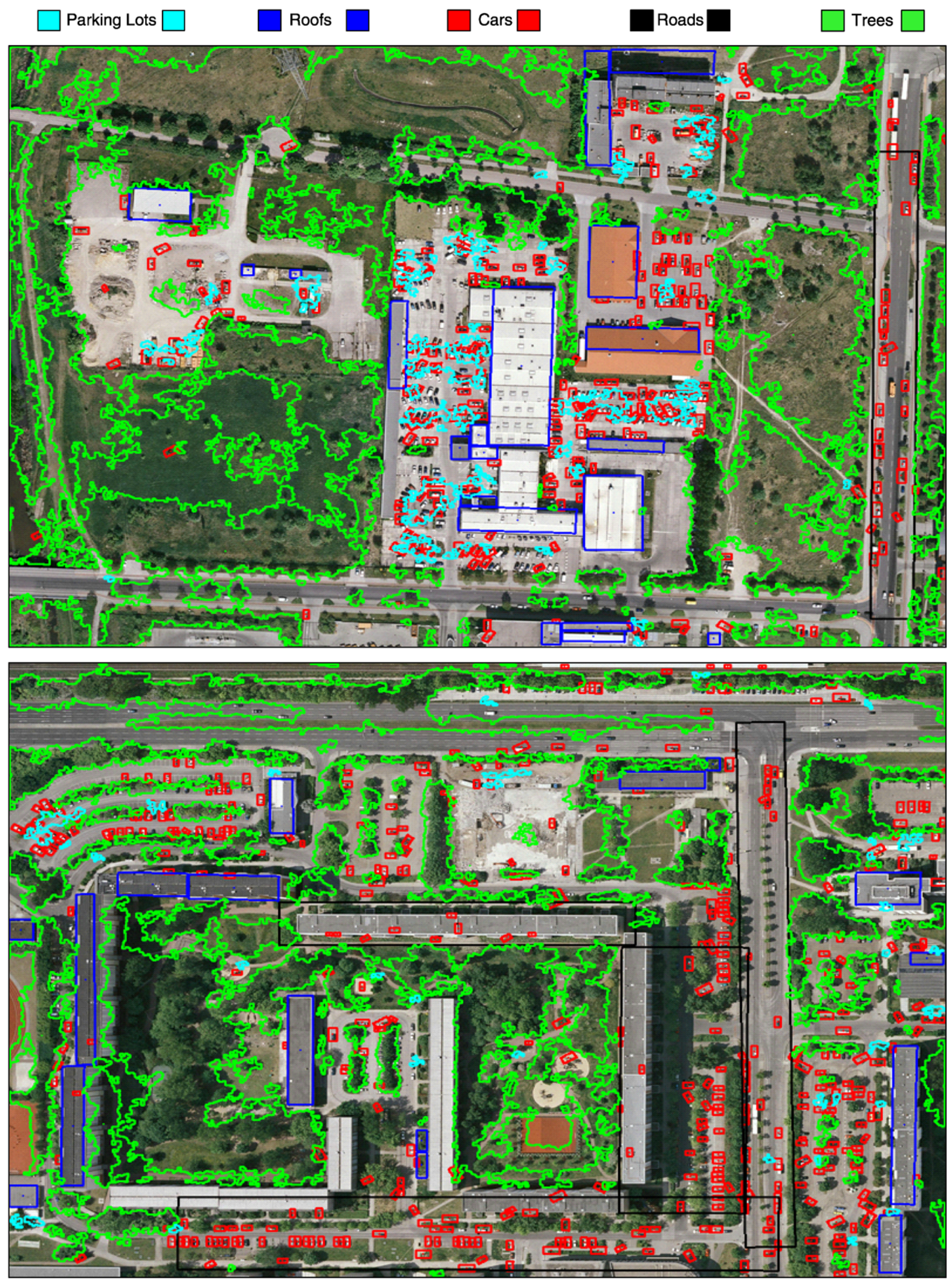

Fig. 27 Flat configurations of parsed images 
another. The fully learned model does not make these mistakes. While $\varepsilon_{1}$ is definitely variable, we strive to select a value that produces good results while still minimizing the size of our relation set $R$.

Figures 26 and 27 show the final results of our algorithm on a number of other urban aerial images. We used our algorithm to find the best parse graph representations for each object and here just display the flattened configurations of the highest probability parse graph for each scene. We can see that the majority of objects are detected accurately, though there are still a few false positives.

We would like to compare our methods to other works in the field, but, as mentioned in Sect. 1, we are hard-pressed to find competing algorithms that identify multiple categories of objects. Similarly it was quite difficult to find benchmarks on consistent datasets in the aerial imaging community, so we would like to offer these results as a benchmark on the aerial images we used from the Lotus Hill Database. These images will be available from the Lotus Hill Institute's website (http://www.imageparsing.com) and can be used freely by anyone else interested in testing on them.

\section{Discussion and Future Work}

In this paper we presented a 3-level contextual hierarchy for modeling aerial images that automatically augments a hierarchical model with relational constraints using a minimax entropy framework. The learning algorithm was able to iteratively add relationships from even large dictionaries of potential relationships in order to model the statistics of the aerial images. We found that this learning process was impeded only by the time needed to sample new images, which can be made tractable through a number of optimizations. In the end our model selected only a small subset of the dictionary of relationships, yet was still able to accurately recreate aerial images. The current work relied on hand-labeled data, but we would like to relax this assumption to first do unsupervised recognition of object types before learning the scene structure in future work.

The bottom-up methods we used along with our threephase Bayesian inference algorithm were instrumental in obtaining the results that we did. Using $\mathrm{C} 4$ to swap interpretations of the scene was extremely helpful, and at times critical, in finding a good subset of detections to represent the scene. Our top-down prediction did not, however, significantly improve results much, as our algorithm only proposed objects that met the line grammar relationship rules (e.g. cars). As was seen in Fig. 21, very few cars are actually added to the representation. This work served as a proof of concept that some combined bottom-up/top-down inference can definitely improve performance, but in this case there were so many objects in each scene that an improvement by 10-20 newly detected objects didn't significantly impact our classification rate. For specific tasks, for example one in which finding every car is important, this technique will be very useful.

In our experiments we hand-defined our 3-level hierarchy and added the context automatically. When learning the hierarchy automatically, however, the question remains as to when one uses hierarchy to group objects and when one simply uses context. We found that grouping objects made our representation simpler and allowed us to avoid the computational inefficiency of computing a fully pairwise graph between all objects. However, we certainly could have created a flat model and added constraints to that. The question of when to use hierarchy and when context on a flat model suffices is an interesting and unresolved one.

Overall we found that our method was able to learn relationships flexibly and out-performs commonly-used singleobject detectors. We hope in the future to find a combination of initial detectors, improved bottom-down inference, and adjustments to $\mathrm{C} 4$ that will improve our results even further.

Acknowledgements This work at UCLA is supported by an NSF grant IIS-0713652 and ONR grant N00014-05-01-0543. The work at Lotus Hill Research Institute is supported by 863 project 2008AA01Z126 and NSFC grant 60776793. We thank the Lotus Hill Dataset for groundtruth annotation (Yao et al. 2007) for the aerial images used in this paper and Benjamin Yao for his many helpful discussions and his assistance with preparing the dataset.

Open Access This article is distributed under the terms of the Creative Commons Attribution Noncommercial License which permits any noncommercial use, distribution, and reproduction in any medium, provided the original author(s) and source are credited.

\section{References}

Barbu, A., \& Zhu, S.-C. (2005). Generalizing Swendsen-Wang to sampling arbitrary posterior probabilities. Pattern Analysis and Machine Intelligence, 27, 1239-1253.

Berg, A., Grabler, F., \& Malik, J. (2007). Parsing images of architectural scenes. In IEEE 11th international conference on computer vision.

Chen, H., Xu, Z., Liu, Z., \& Zhu, S.-C. (2006). Composite templates for cloth modeling and sketching. In Proceedings of the IEEE conference on computer vision and pattern recognition (Vol. 1, pp. 943-950).

Chi, Z., \& Geman, S. (1998). Estimation of probabilistic context-free grammars. Computational Linguistics, 24(2).

Felzenszwalb, P., \& Huttenlocher, D. (2005). Pictorial structures for object recognition. International Journal of Computer Vision, 61(1), 55-79.

Fischler, M., \& Elschlager, R. (1973). The representation and matching of pictorial structures. IEEE Transactions on Computers, 22(1), 67-92.

Freund, Y., \& Schapire, R. (1997). A decision-theoretic generalization of on-line learning and an application to boosting. Journal of Computer and System Sciences, 55.

$\mathrm{Fu}, \mathrm{K}$. S. (1981). Syntactic pattern recognition and applications. New York: Prentice Hall. 
Han, F., \& Zhu, S.-C. (2005). Bottom-up and top-down image parsing by attribute graph grammar. In Proceedings of the international conference on computer vision (Vol. 2).

Hinz, S., \& Baumgartner, A. (2000). Road extraction in urban areas supported by context objects. International Archives of Photogrammetry and Remote Sensing, 33.

Jin, Y., \& Geman, S. (2006). Context and hierarchy in a probabilistic image model. In Proceedings of the IEEE conference on computer vision and pattern recognition (Vol. 2, pp. 2145-2152).

Johnson, M., Geman, S., Canon, S., Chi, Z., \& Riezler, S. (1999). Estimators for stochastic unification-based grammars. In Proceedings ACL'99, Maryland.

Keselman, Y., \& Dickinson, S. (2001). Generic model abstraction from examples. Pattern Analysis and Machine Intelligence, 27, 11411156.

Li, F.-F., \& Perona, P. (2005). A bayesian hierarchical model for learning natural scene categories. In Proceedings of the IEEE conference on computer vision and pattern recognition (Vol. 2, pp. 524531).

Li, Y., Atmosukarto, I., Kobashi, M., Yuen, J., \& Shapiro, L. (2005). Object and event recognition for aerial surveillance. In SPIE - the international society for optical engineering.

Maloof, M. A., Langley, P., Binford, T. O., Nevatia, R., \& Sage, S. (2003). Improved rooftop detection in aerial images with machine learning. Machine Learning.

Matsuyama, T., \& Hang, V. (1990). Sigma: A framework for image understanding integration of bottom-up and top-down analyses. New York: Plenum.

Moissinac, H., Matre, H., \& Bloch, I. (1994). Urban aerial image understanding using symbolic data. In Image and signal processing for remote sensing, proc. SPIE.

Nicolas, B., Viglino, J., \& Cocquerez, J. (2000). Knowledge based system for the automatic extraction of road intersections from aerial images. International Archives of Photogrammetry and Remote Sensing.

Ohta, Y. (1985). Knowledge-based interpretation of outdoor natural color scenes. London: Pitman.

Porway, J., \& Zhu, S. C. (2009). C4: Stochastic inference on graphical models with positive and negative edges for rapidly exploring competing solutions (Technical Report).

Porway, J., Wang, K., Yao, B., \& Zhu, S.-C. (2008). A hierarchical and contextual model for aerial image understanding. In Proceedings of the IEEE conference on computer vision and pattern recognition.

Shotton, J., Winn, J., Rother, C., \& Criminisi, A. (2006). Textonboost: Joint appearance, shape and context modeling for multi-class object recognition and segmentation. In Proceedings of the European conference on computer vision (pp. 1-15).

Siddiqi, K., Shokoufandeh, A., Dickinson, S., \& Zucker, S. W. (1999). Shock graphs and shape matching. International Journal of Computer Vision, 35(1), 13-32.

Singhal, A., Luo, J., \& Zhu, W. (2003). Probabilistic spatial context models for scene content understanding. In IEEE computer society conference on computer vision and pattern recognition (Vol. 1).
Sivic, J., Russell, B., Efros, A., Zisserman, A., \& Freeman, W. (2005). Discovering objects and their location in images. In Tenth IEEE international conference on computer vision.

Sudderth, E. B., Torralba, A., Freeman, W. T., \& Willsky, A. S. (2005). Describing visual scenes using transformed Dirichlet processes. In Neural information processing systems.

Swendsen, R., \& Wang, J. (1987). Nonuniversal critical dynamics in Monte Carlo simulations. Physical Review Letters.

Todorovic, S., \& Ahuja, N. (2006). Extracting subimages of an unknown category from a set of images. In Proceedings of the IEEE conference on computer vision and pattern recognition (Vol. 1, pp. 927-934).

Tu, Z., \& Zhu, S.-C. (2002). Image segmentation by data-driven Markov chain Monte Carlo. IEEE Transactions on Pattern Analysis and Machine Learning, 24(5), 657-673.

Ullman, S., Sali, E., \& Vidal, M. (2001). A fragment-based approach to object representation and classification. In Proceedings of the 4th international workshop on visual form.

Vestri, C., \& Devernay, F. (2001). Using robust methods for automatic extraction of buildings. In Proceedings of the IEEE conference on computer vision and pattern recognition (Vol. 1).

Viola, P., \& Jones, M. (2001). Rapid object detection using a boosted cascade of simple features. In Proceedings of the IEEE conference on computer vision and pattern recognition (pp. 511-518).

Wainwright, M., \& Jordan, M. (2008). Graphical models, exponential families, and variational inference. Foundations and Trends in Machine Learning, 1(1), 1-305.

Weber, M., Welling, M., \& Perona, P. (2000). Towards automatic discovery of object categories. In Proceedings of the IEEE conference on computer vision and pattern recognition (Vol. 2, pp. 101108).

Wei, L., \& Prinet, V. (2005). Building detection from high-resolution satellite image using probability model. In Geoscience and remote sensing symposium, IGARSS (pp. 25-29).

Wu, T. F., Xia, G. S., \& Zhu, S.-C. (2007). Compositional boosting for computing hierarchical image structures. In Proceedings of the IEEE conference on computer vision and pattern recognition (pp. 1-8).

Yao, B., Yang, X., \& Zhu, S.-C. (2007). Introduction to a large scale general purpose groundtruth dataset: methodology, annotation tool, and benchmarks. Energy Minimization Methods in Computer Vision and Pattern Recognition, 4697, 169-183.

Zhao, T., \& Nevatia, R. (2001). Car detection in low resolution aerial image. In IEEE international conference on computer vision (Vol. 1).

Zhu, S.-C., \& Mumford, D. (2006). A stochastic grammar of images. Foundation and Trends in Computer Graphics and Vision, 2(4), 259-362.

Zhu, S.-C., Wu, Y.-N., \& Mumford, D. (1998). Frame: Filters, random fields, and minimax entropy towards a unified theory for texture modeling. International Journal of Computer Vision, 2, 107-126.

Zhu, L., Lin, C., Huang, H., Chen, Y., \& Yuille, A. (2008). Unsupervised structure learning: Hierarchical recursive composition, suspicious coincidence and competitive exclusion. In Proceedings of the 10th European conference on computer vision: Part II. 